\title{
Impuesto al patrimonio: una propuesta tributaria deficiente
}

\author{
Hermann González B., ${ }^{a}$ Felipe Larraín B., ${ }^{\text {a,b }}$ Óscar Perelló P., ca \\ a Centro Latinoamericano de Políticas Económicas y Sociales, Clapes UC, Chile \\ b Pontificia Universidad Católica de Chile, Chile \\ c University College London, Reino Unido
}

\begin{abstract}
RESUMEN: Este trabajo analiza la conveniencia de implementar un impuesto al patrimonio en Chile. En primer lugar, se presenta el contexto tributario y económico en que se desarrolla la discusión sobre el impuesto al patrimonio. Luego, se revisan los efectos macroeconómicos y fiscales de establecer este tipo de impuesto. La teoría económica sugiere que un impuesto al patrimonio puede inducir salidas de capitales, un menor retorno del capital, una reducción del precio de los activos gravados y costos de eficiencia para el Estado. En esa línea, la
\end{abstract}

\footnotetext{
Herman González es MA en Economía, Pontificia Universidad Católica de Chile. Coordinador macroeconómico del Centro Latinoamericano de Políticas Económicas y Sociales, Pontificia Universidad Católica de Chile (Clapes UC). Dirección: Avda. Libertador Bernardo O'Higgins 440, Santiago Centro, Santiago, Chile, CP 8331010. Email: hegonzal@uc.cl.

Felipe Larraín es PhD en Economía, Harvard University, EEUU. Profesor titular de la Facultad de Economía y Administración, Pontificia Universidad Católica de Chile, y miembro del Comité Ejecutivo del Centro Latinoamericano de Políticas Económicas y Sociales, Pontificia Universidad Católica de Chile (Clapes UC). Dirección: Avda. Libertador Bernardo O'Higgins 440, Santiago Centro, Santiago, Chile, CP 8331010. Email: flarrainb@uc.cl.

Óscar Pereloó es MA en Economía, Pontificia Universidad Católica de Chile, y estudiante de doctorado del Departamento de Economía, University College London (UCL). Investigador externo del Centro Latinoamericano de Políticas Económicas y Sociales, Pontificia Universidad Católica de Chile (Clapes UC). Dirección: Avda Libertador Bernardo O'Higgins 440, Santiago Centro, Santiago, Chile, CP 8331010 . Email: oiperell@uc.cl.
}

Agradecemos los valiosos comentarios y sugerencias de dos árbitros anónimos y la asistencia en investigación de Patricio Mansilla. 
experiencia de los países de la OCDE muestra que el impuesto al patrimonio disminuye la riqueza reportada por los hogares, mientras que estimaciones empíricas para la elasticidad del stock de capital sugieren que sus efectos sobre la actividad económica son relevantes. Por otro lado, los efectos sobre la distribución del ingreso de este mecanismo son acotados. En términos de recaudación fiscal, la experiencia de los países de la OCDE sugiere una baja contribución del impuesto al patrimonio a las arcas fiscales. Finalmente, se evalúa la propuesta de impuesto al patrimonio presentada para Chile y se argumenta que la recaudación fiscal sería muy inferior a lo estimado por los promotores de esta iniciativa.

Palabras Clave: impuestos, patrimonio, riqueza, política tributaria, stock de capital

RECIBIDO: octubre 2020 / ACEPTADO: enero 2021

\section{Wealth Tax: A Flawed Proposal}

ABSTRACT: This paper analyzes the convenience of implementing a wealth tax in Chile. First, we present the current tax and economic context in which the discussion on the wealth tax takes place. Then, we review the macroeconomic and fiscal effects of this type of tax. Economic theory suggests that a wealth tax induces capital outflows, a lower return on capital, a reduction in the price of taxed assets, and efficiency costs for the Government. Moreover, the experience of OECD countries shows that the wealth tax decreases self-reported household wealth, while empirical estimates for the capital stock elasticity suggest that its effects on economic activity are sizable. On the other hand, the effects on income distribution are limited. In terms of tax collection, the recent experience of OECD countries shows a low contribution of the wealth tax to the fiscal budget. Finally, we assess the wealth tax proposal presented for Chile and argue that tax collection would be much lower than estimated by the sponsors of this policy.

KEYWORDS: taxes, wealth, wealth tax, tax policy, capital stock

ReCEIvED: October 2020 / AcCEPTED: January 2021

ste documento analiza la conveniencia de implementar un impuesto al patrimonio (o impuesto a la riqueza personal neta) en Chile, considerando aspectos macroeconómicos y fiscales. Si bien la crisis económica generada por el Covid-19 ha reimpulsado el debate sobre el impuesto al patrimonio a nivel global, la tendencia seguida por la mayoría de los países de la OCDE durante las últimas décadas fue eliminar este tipo de impuestos. Considerando la teoría económica, la experiencia 
de los países de la OCDE que han implementado este tipo de impuestos y estimaciones recientes para la elasticidad del stock de capital, argumentamos que el impuesto al patrimonio tendría un impacto negativo y significativo sobre la actividad económica. Lo anterior es particularmente contraproducente en el contexto económico actual, donde uno de los principales desafíos que enfrenta el país es la recuperación de los empleos perdidos durante la crisis sanitaria. Por otro lado, la experiencia internacional sugiere que la recaudación fiscal sería inferior a lo estimado en la propuesta tributaria presentada para Chile (Boletín $N^{\circ}$ 13555-07).

En primer lugar, se analiza el contexto tributario y económico en el que se desarrolla la discusión sobre el impuesto al patrimonio. Este mecanismo fue discutido institucionalmente en la negociación entre gobierno y oposición a fines de 2019. En ese entonces, ante los problemas técnicos asociados a este impuesto, se optó por implementar otras medidas que permitieran obtener recursos fiscales de forma progresiva, como fue el nuevo tramo del impuesto a la renta y las mayores contribuciones de bienes raíces incorporados en la Ley $N^{\circ} 21.210$. Por otro lado, la propuesta de impuesto al patrimonio se ha realizado mediante una Moción Parlamentaria que está fuera del marco constitucional para la política tributaria, tensionando el orden institucional. En cuanto al contexto económico, se debe considerar que la discusión tributaria se desarrolla mientras el país atraviesa su peor momento económico desde la crisis de 1982.

En cuanto a los efectos macroeconómicos, la teoría económica sugiere que el impuesto al patrimonio genera un impacto adverso sobre la economía a través de diversos canales de transmisión. Primero, en un mundo altamente globalizado, el impuesto al patrimonio tiende a inducir salidas de capitales que repercuten sobre la inversión y la capacidad productiva de la economía. Segundo, el impuesto al patrimonio es, en la práctica, un impuesto sobre el retorno del capital, lo que disminuye los incentivos al ahorro y la inversión. En esta línea, cabe señalar que un impuesto relativamente pequeño sobre el patrimonio representa un impuesto de gran magnitud sobre el retorno del capital. Tercero, el impuesto al patrimonio puede disminuir el precio de los activos gravados, lo que no solo afectaría a los contribuyentes de alto patrimonio, sino que a todos los hogares y empresas que cuenten con tales activos. Cuarto, el impuesto al patrimonio conlleva altos costos de eficiencia para el Estado en términos de avaluación de activos. 
Este trabajo también revisa un conjunto de estudios empíricos recientes en países de la OCDE. La evidencia analizada para Colombia, Dinamarca, España, Holanda, Suecia y Suiza sugiere que el impuesto al patrimonio disminuye la riqueza de los hogares. En el caso de Suiza, es posible descomponer este efecto en sus distintos canales de transmisión. En particular, cerca de la mitad del efecto se explicaría por movimientos de capitales, caídas en el precio de activos y menores tasas de ahorro, mientras que el resto del efecto respondería a prácticas de elusión y evasión tributaria, entre otros factores. Por otro lado, la evidencia analizada para Francia sugiere que la salidas de capitales inducidas por el impuesto al patrimonio son significativas.

El orden de magnitud de los efectos del impuesto al patrimonio sobre la economía dependerá de la elasticidad de largo plazo del stock de capital que tengan los contribuyentes gravados por el impuesto. Si esta elasticidad es alta, incluso un impuesto a la riqueza aparentemente pequeño puede tener implicancias significativas sobre la actividad económica. Considerando las elasticidades estimadas por la literatura económica, se argumenta que los efectos de un impuesto al patrimonio sobre la actividad económica son relevantes. Por otro lado, los efectos sobre la distribución del ingreso de este mecanismo son acotados. Lo anterior es particularmente cierto para el caso de Chile, dado que la principal brecha con la OCDE está en el efecto de las transferencias sociales sobre la distribución del ingreso, mientras que el margen para mejorar la distribución del ingreso a través de impuestos es significativamente menor.

En términos de recaudación fiscal, la experiencia de los países de la OCDE es concluyente respecto de la baja efectividad del impuesto al patrimonio. Desde 1990 a la fecha, la recaudación promedio de los países OCDE que han utilizado este impuesto es de solo $0,17 \%$ del PIB $(0,51 \%$ de la recaudación tributaria total). Al desagregar a nivel de países, la mayoría de los países de la OCDE que ha utilizado un impuesto al patrimonio ha recaudado menos de $0,2 \%$ del PIB, mientras que solo dos países han logrado recaudar más de $0,5 \%$ del PIB.

Finalmente, se analiza la propuesta de impuesto al patrimonio para Chile (Boletín N¹3555-07). Esta propuesta contiene cuatro diferencias técnicas respecto del impuesto al patrimonio aplicado en otros países de la OCDE. Primero, la propuesta para Chile utiliza el concepto de patrimonio 'bruto', lo que difiere de la experiencia de los países OCDE y de la con- 
ceptualización económica de la riqueza personal, donde esta se considera 'neta' de deudas. Segundo, la propuesta plantea que el impuesto se cobraría por una sola vez, mientras que en décadas recientes los países de la OCDE han utilizado impuestos que se cobran de manera regular. Sin embargo, la experiencia reciente de España, donde este impuesto fue instaurado de manera transitoria para enfrentar la crisis del Euro en 2011 y se mantiene vigente hasta la fecha, sugiere que la promesa de un impuesto transitorio es poco creíble. Tercero, la tasa impositiva propuesta para Chile $(2,5 \%)$ es superior a las tasas observadas en la mayoría de las experiencias de países OCDE. Cuarto, la propuesta actual considera un umbral discreto para los patrimonios (US\$22 millones), lo que refuerza los incentivos al subreporte de activos.

Respecto de la estimación fiscal que acompaña a la propuesta de impuesto al patrimonio para Chile, se observa que esta difiere de la experiencia de los países de la OCDE. En particular, la Moción Parlamentaria plantea recaudar US\$6.500 millones (2,3\% del PIB), lo que es mayor a la recaudación fiscal obtenida por cualquiera de los países de la OCDE en décadas recientes. Al considerar la recaudación fiscal por cada punto de impuesto al patrimonio, la propuesta para Chile espera recaudar $0,92 \%$ del PIB por cada punto de impuesto, lo que es muy superior a lo recaudado por Noruega $(0,53 \%)$, Holanda $(0,26 \%)$, Islandia $(0,2 \%)$, Austria $(0,14 \%)$, Suecia $(0,13 \%)$, Alemania $(0,11 \%)$ y Finlandia $(0,1 \%)$ por cada punto de impuesto.

Este artículo extiende otros trabajos sobre el tema en al menos tres dimensiones. ${ }^{1}$ En primer lugar, además de proporcionar argumentos teóricos adicionales, se presenta una revisión empírica de los efectos macroeconómicos del impuesto al patrimonio en los países de la OCDE que lo han implementado. Segundo, se discuten las estimaciones realizadas para la elasticidad del stock de capital en la literatura económica, las que son fundamentales para evaluar la magnitud de los efectos macroeconómicos antes mencionados. Tercero, se analiza la propuesta de impuesto al patrimonio presentada para Chile, abordando sus principales características técnicas y su estimación fiscal. El resto de este documento se estructura como sigue. La sección 1 presenta el contexto tributario y económico. La sección 2 analiza los efectos macroeconómicos del impuesto

\footnotetext{
${ }^{1}$ Ver, por ejemplo, Cordero y Vergara (2020).
} 
al patrimonio. La sección 3 analiza el impacto sobre la recaudación fiscal. La sección 4 evalúa la propuesta presentada para el caso de Chile. La sección 5 concluye.

\section{Contexto tributario y económico en Chile}

\section{Acuerdos tributarios recientes}

La discusión sobre el impuesto al patrimonio no es nueva, sino que tiene antecedentes recientes en la crisis social ocurrida en Chile a partir de octubre de 2019. Tales hechos se produjeron en momentos en que se discutía el proyecto de modernización tributaria en el Senado, después de haber pasado con éxito el primer trámite de la Cámara de Diputados, fruto de un acuerdo entre el gobierno, el Partido Demócrata Cristiano, sectores independientes y las pymes. Dicho acuerdo consideraba la inclusión de medidas que aumentarían la recaudación de forma progresiva.

La discusión tributaria que continuó en el Senado, como muchas otras discusiones que se producían en ese momento en nuestro país, estuvo influenciada por la crisis social que atravesaba el país. La oposición planteaba que la causa del malestar social radicaba en la desigualdad de ingresos, de manera que la solución requería que quienes tuvieran más recursos, aportaran más. En este contexto, representantes políticos de gobierno y de oposición discutieron la posibilidad de crear un impuesto al patrimonio o a las grandes fortunas, representadas por el $1 \%$ más rico de nuestro país. ${ }^{2}$ Sin embargo, atendiendo los problemas técnicos que tienen los impuestos al patrimonio, los que llevaron a su eliminación en la mayor parte de los países de la OCDE, la negociación entre el gobierno y la Comisión de Hacienda del Senado optó por una forma distinta de gravar a quienes tienen mayores recursos económicos en Chile.

En primer lugar, se creó un nuevo tramo del impuesto a la renta que considera una tasa de $40 \%$ para los contribuyentes de altos ingresos. Esta tasa afecta a los trabajadores dependientes con rentas mensuales superiores a 15,4 millones de pesos (Impuesto de Segunda Categoría) y a los

\footnotetext{
${ }^{2}$ Ver, por ejemplo: "RN y oposición inician diálogo para impulsar impuesto al 1\% 'más rico'". Disponible en: https://www.latercera.com/politica/noticia/rn-oposicion-inician-dialogoimpulsar-impuesto-al-1-mas-rico/876933/ [20 de octubre 2020].
} 
contribuyentes con rentas anuales por sobre 185 millones de pesos (Impuesto Global Complementario). En el acuerdo firmado entre el gobierno y los senadores se señaló que este nuevo tramo gravaría al 1\% de los contribuyentes de mayores ingresos. En segundo término, atendiendo a que uno de los problemas del impuesto al patrimonio es la movilidad de los capitales, se optó por subir el impuesto al activo inmóvil, es decir, las contribuciones de bienes raíces. Específicamente, se creó una sobretasa del impuesto territorial para activos inmobiliarios de alto valor. La base de esta sobretasa se estima con la suma de los avalúos fiscales de cada contribuyente y tiene un tramo exento de 670 UTA. Al primer tramo, entre 670 y 1.175 UTA, se aplica una tasa de 0,075\%; al segundo tramo, entre 1.175 y 1.510 UTA, una tasa de $0,15 \%$; y al último tramo, por sobre 1.510 UTA, una tasa de $0,275 \%$. En el caso de las personas, este impuesto afectaría a un total 35 mil contribuyentes de alto patrimonio y se estimó que, del total recaudado, un $80 \%$ provendrá del $0,1 \%$ de los contribuyentes de mayores recursos, convirtiéndolo en un impuesto altamente progresivo.

El acuerdo firmado entre el gobierno y el Senado contempló la implementación de dos impuestos que implican un aumento de la carga tributaria para las personas de mayores ingresos de nuestro país. De esta forma, se debe tener en consideración que la alternativa de un impuesto al patrimonio fue discutida hace pocos meses y que la respuesta a este debate, proveniente de la negociación entre el gobierno y el Senado, fue implementar medidas alternativas que se consideraron más efectivas para incrementar la recaudación fiscal de forma progresiva.

\section{Política tributaria dentro del marco constitucional}

Los impuestos, al igual que los proyectos que erogan gasto público, son iniciativa exclusiva del presidente de la República de acuerdo con la Constitución Política del Estado. En particular, el artículo 65 de la Constitución señala que "corresponderá al Presidente de la República la iniciativa exclusiva para imponer, suprimir, reducir o condonar tributos de cualquier clase o naturaleza, establecer exenciones o modificar las existentes y determinar su forma, proporcionalidad o progresión". De esta forma, no corresponde que sea el Congreso el que proponga la creación de un impuesto, como ha sido el caso con la Moción Parlamentaria que propone 
implementar un impuesto al patrimonio, ${ }^{3}$ y hacerlo tensiona aún más el orden institucional vigente.

También va en contra de nuestro orden institucional que el proyecto destine los recursos que recaudaría este impuesto a un fin específico, como es el financiamiento de una renta básica de emergencia. El artículo 19 inciso tercero de la Carta Fundamental establece que "los tributos que se recauden, cualquiera que sea su naturaleza, ingresarán al patrimonio de la Nación y no podrán estar afectos a un destino determinado". Si bien la Constitución permite excepciones al principio de no afectación, este no es el caso del impuesto al patrimonio. En particular, la ley autoriza que determinados tributos puedan estar afectados a fines propios de la defensa nacional. Asimismo, se autoriza que los tributos que gravan actividades o bienes que tengan una clara identificación regional o local puedan ser aplicados por las autoridades regionales o comunales para el financiamiento de obras de desarrollo (Art. 19, numeral 20).

La idea de crear un impuesto al patrimonio mediante una Moción Parlamentaria no solo tensiona el orden institucional, sino que afecta la imagen externa de nuestro país en términos macroeconómicos. Esto, en la práctica, puede acelerar el deterioro de la clasificación crediticia soberana $y$, finalmente, generar peores condiciones de acceso al financiamiento para el Estado, las empresas y las familias. De hecho, a raíz del recientemente aprobado proyecto de retiro de parte de los fondos de las cuentas individuales de las AFP, que también tensionó la institucionalidad, la agencia clasificadora de riesgo Moody's señaló que "es un evento que puede sentar un mal precedente desde el punto de vista crediticio soberano". El informe de la agencia criticó que la propuesta viniese del Congreso y no del ejecutivo, así como también la ausencia de un análisis técnico que los respaldara. ${ }^{4}$

\section{Sistema tributario en Chile}

Si bien este documento se enfoca en evaluar los efectos macroeconómicos y fiscales de un impuesto al patrimonio, es importante considerar

\footnotetext{
${ }^{3}$ Ver Moción Parlamentaria correspondiente al Boletín N ${ }^{\circ}$ 13555-07. Disponible en: https:// www.camara.cl/verDoc.aspx?prmID=13876\&prmTIPO=INICIATIVA [20 de octubre 2020].

${ }^{4}$ Ver "Moody's y retiro del 10\%: 'Puede sentar un mal precedente desde el punto de vista crediticio soberano'". Disponible en: https://www.latercera.com/pulso/noticia/moodys-yretiro-del-10-puede-sentar-un-mal-precedente-desde-el-punto-de-vista-crediticio-soberano/QCYN33ACWBHYZBBPDAYQIYP2PM/ [24 de julio 2020].
} 
que este análisis se inserta en un debate más amplio, el que incluye al sistema tributario en su conjunto. En octubre de 2020, el Ministerio de Hacienda conformó la Comisión Tributaria para el Crecimiento y la Equidad, cuyo objetivo es analizar la dirección en la que debe avanzar el sistema tributario chileno en el mediano plazo. ${ }^{5}$ Para definir este horizonte, es ilustrativo comparar la recaudación fiscal por tipo de impuesto en Chile y en los países de la OCDE, de manera de identificar en qué tributos se encuentran las principales diferencias. En el marco de nuestro análisis, interesa saber si tales diferencias radican en impuestos a la propiedad, entre los que se incluye el impuesto al patrimonio, o en otras categorías de impuestos.

El Gráfico 1 presenta la recaudación tributaria por tipo de impuesto para el caso de Chile, comparándola con la mediana de los países de la OCDE. Las cifras de recaudación se expresan como porcentaje del PIB, de manera de analizar su magnitud respecto del tamaño de la economía.

En primer lugar, se observa que las principales diferencias entre Chile y la OCDE se encuentran en el impuesto a la renta y en las contribuciones de seguridad social. Sin embargo, la diferencia en las contribuciones de seguridad social se explica, en buena medida, porque en Chile la mayor parte de estas se realizan a través de aportes a organismos privados (AFPs e isapres) y no mediante impuestos propiamente tales. En particular, en Chile, un $20 \%$ de estas contribuciones se realiza a través de impuestos, mientras que la mediana de los países de la OCDE es un 97\%. ${ }^{6}$ En el caso del impuesto a la renta, la diferencia está incidida por el hecho de que el tramo exento es significativamente más amplio en el caso de $\mathrm{Chile}^{7}$ y por la naturaleza semiintegrada del sistema tributario.

En cuanto a los impuestos sobre la propiedad, estos son relativamente similares en Chile y la OCDE, representando un 1,1\% y un 1,5\% del PIB, respectivamente. De esta forma, no es posible argumentar que una de las principales diferencias entre la recaudación tributaria de Chile

\footnotetext{
${ }^{5}$ Para más información, ver plataforma web del Ministerio de Hacienda. Disponible en: https://www.hacienda.cl/noticias-y-eventos/noticias/ministerio-de-hacienda-crea-comision-tributaria-para-el-crecimiento-y-la [14 de enero 2021].

${ }^{6}$ Ver OECD Stats (OCDE 2020).

${ }^{7}$ A modo de ejemplo, en países como Nueva Zelanda y Corea del Sur no existe un tramo exento propiamente tal; los contribuyentes de menores ingresos pagan una tasa marginal de $10,5 \%$ y $6 \%$, respectivamente. Por otro lado, en Chile, los contribuyentes con una renta mensual de hasta $\$ 690.000$ están exentos de este tipo de impuesto.
} 
Gráfico 1. RECAUDACIÓN TRIBUTARIA POR TIPO DE IMPUESTO EN CHILE Y LA OCDE (\% DEL PIB)

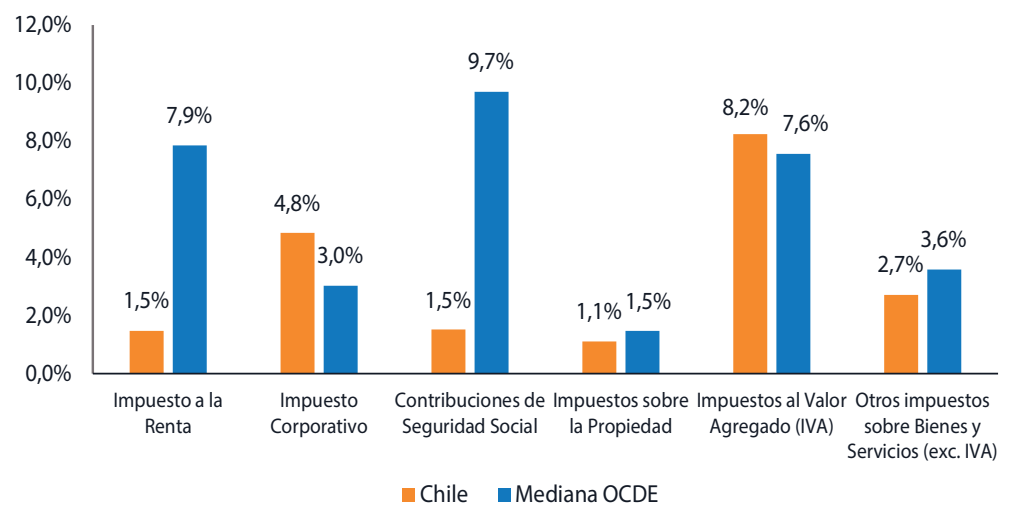

Fuente: Elaboración propia en base a OCDE (2020).

y los países de la OCDE radica en los impuestos sobre la propiedad, lo que podría utilizarse para justificar la implementación de un impuesto al patrimonio. Por otro lado, cabe señalar que en Chile la recaudación por concepto de impuesto corporativo está por encima de la mediana de la $\mathrm{OCDE}$, lo que afecta directamente al retorno del capital. Lo anterior es relevante para nuestro análisis dado que, en la práctica, el impuesto al patrimonio representa un impuesto adicional sobre el retorno del capital, lo que se analizará con mayor detalle bajo el subtítulo 'Principales efectos macroeconómicos'.

\section{Crisis económica}

En 2020, Chile experimentó su mayor recesión desde la recesión económica de 1982, como consecuencia de las dos crisis que hemos enfrentado en menos de un año: la crisis social iniciada en octubre de 2019 y la pandemia del Covid-19, que afecta la economía desde marzo de 2020. De acuerdo con estimaciones del Banco Central, el PIB se habría contraído en torno a $-6,0 \%$ en $2020 .{ }^{8}$ Esta caída es muy superior a lo observado durante la crisis asiática de 1999, cuando el PIB se contrajo $-0,4 \%$, y du-

\footnotetext{
${ }^{8}$ El rango de proyección es de $-6,25 \%$ a $-5,75 \%$, de acuerdo con el Informe de Política Monetaria de diciembre, Banco Central de Chile (2020b).
} 
rante la crisis subprime de 2009, en que el PIB disminuyó un -1,6\% (ver Gráfico 2). Por su parte, se estima que la inversión habría retrocedido cerca de $-13,0 \%$ en 2020. El año 2021 veremos solo una recuperación parcial de la inversión y el producto, lo que a su vez está sujeto a un alto grado de incertidumbre en torno al desarrollo de la pandemia y la reaparición de los actos de violencia en el país.

La contracción de la economía ha tenido efectos significativos sobre el mercado laboral. En el peak de la crisis sanitaria (julio de 2020), el número de puestos de trabajo perdidos superó los 1,8 millones en términos interanuales, con lo que el número de ocupados descendió a niveles cercanos a los de inicios de 2010. Por su parte, la tasa de desempleo superó los dos dígitos y alcanzó un 13,1\% en el mismo período. ${ }^{9}$ Cabe señalar que, en un contexto de crisis económica, la tasa de desempleo subestima el nivel de desempleo efectivo debido a la caída de la participación laboral. En esta línea, la tasa de desempleo corregida por la disminución de la participación laboral alcanzó un máximo de 24,4\% en julio de 2020, lo que refleja la verdadera magnitud de los efectos de la crisis sanitaria sobre el mercado laboral. ${ }^{10}$

Si bien el empleo ha comenzado a recuperarse gradualmente en los meses recientes, las cifras actualizadas hasta noviembre de 2020 muestran que aún queda por recuperar más de un millón de puestos de trabajo. De esta forma, una recuperación completa y sostenible del mercado laboral solo será posible en la medida en que la actividad económica recobre su dinamismo. Para este objetivo se requiere un conjunto de medidas que promuevan la inversión nacional y extranjera, mientras que la creación de un impuesto al patrimonio, como se expondrá en las secciones siguientes, es contraria a cualquier estrategia de reactivación económica. ${ }^{11}$

\footnotetext{
${ }^{9}$ Ver INE (2020).

${ }^{10}$ Las tasa de desempleo corregida por participación laboral corresponde a lo estimado por el Centro de Encuestas y Estudios Longitudinales UC (2020), utilizando la Encuesta de Empleo Covid-19. Cabe señalar que, si bien la corrección por participación laboral podría realizarse en cualquier período, solo en tiempos de crisis económica la tasa de desempleo corregida diferirá significativamente de la medición habitual.

${ }^{11}$ Cabe destacar el acuerdo alcanzado entre el gobierno y diversos partidos de oposición a mediados de junio de 2020, que considera un marco fiscal de hasta US\$12 mil millones en un horizonte de 24 meses, incorporando medidas de apoyo para las familias, empresas y municipios, recursos para la salud y un plan de inversión pública. En esa ocasión, nada se mencionó respecto de la creación de un impuesto al patrimonio, comprendiendo la relevancia de implementar medidas que faciliten la recuperación económica.
} 
Gráfico 2. CRECIMIENTO DEL PIB (VAR. \% ANUAL)

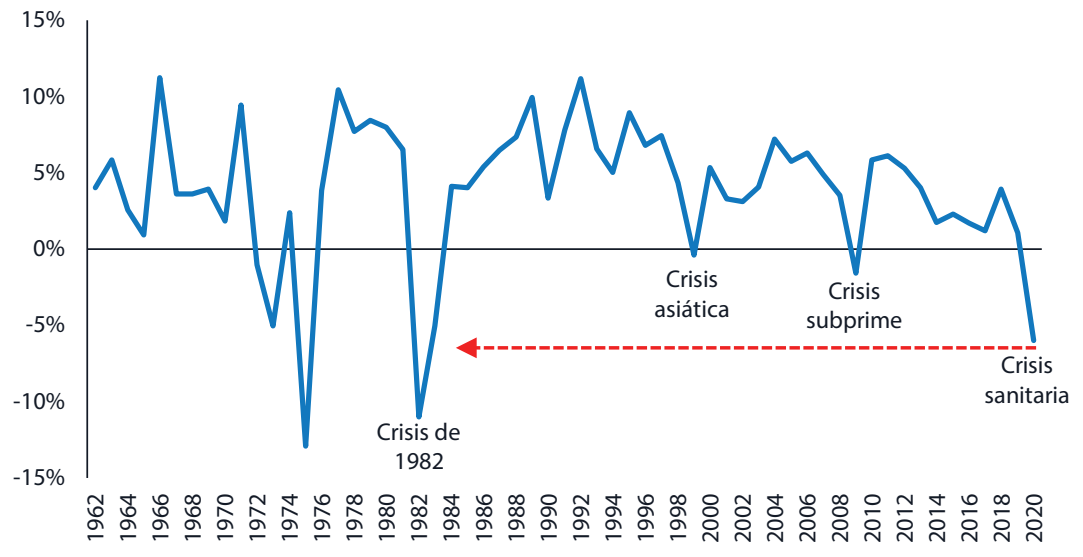

Fuente: Elaboración propia en base a Banco Central de Chile (2020a, 2020b).

\section{Efectos económicos del impuesto al patrimonio}

La tendencia seguida por la mayoría de los países desarrollados ha sido eliminar el impuesto al patrimonio. De acuerdo a los registros oficiales de la OCDE, en 1990 había doce países que contaban con un impuesto al patrimonio, mientras que en 2018 solo tres países usaban este mecanismo de recaudación (ver Gráfico 3). En la actualidad, este número ascendería a cuatro países, considerando la reciente incorporación de Colombia a la OCDE durante 2020. Los otros países de la OCDE que mantienen vigente este tipo de impuesto corresponden a España, Noruega y Suiza. Por su parte, Austria eliminó el impuesto al patrimonio en 1994; Dinamarca y Alemania en 1997; Holanda en 2001; Finlandia, Islandia y Luxemburgo en 2006; Suecia en 2007 y, más recientemente, Francia en 2017. Cabe señalar que algunos países de la OCDE utilizan impuestos sobre el valor de ciertos activos específicos, como es el caso de Bélgica, Holanda e Italia, pero tales tributos no representan un impuesto al patrimonio propiamente tal, de acuerdo con la definición de la OCDE y otros organismos especializados. $^{12}$

\footnotetext{
12 Ver The Role and Design of Net Wealth Taxes in the OECD (OCDE 2018) y Worldwide Estate and Inheritance Tax Guide (Ernst \& Young 2019).
} 
Gráfico 3. NÚMERO DE PAÍSES DE LA OCDE CON IMPUESTO AL PATRIMONIO

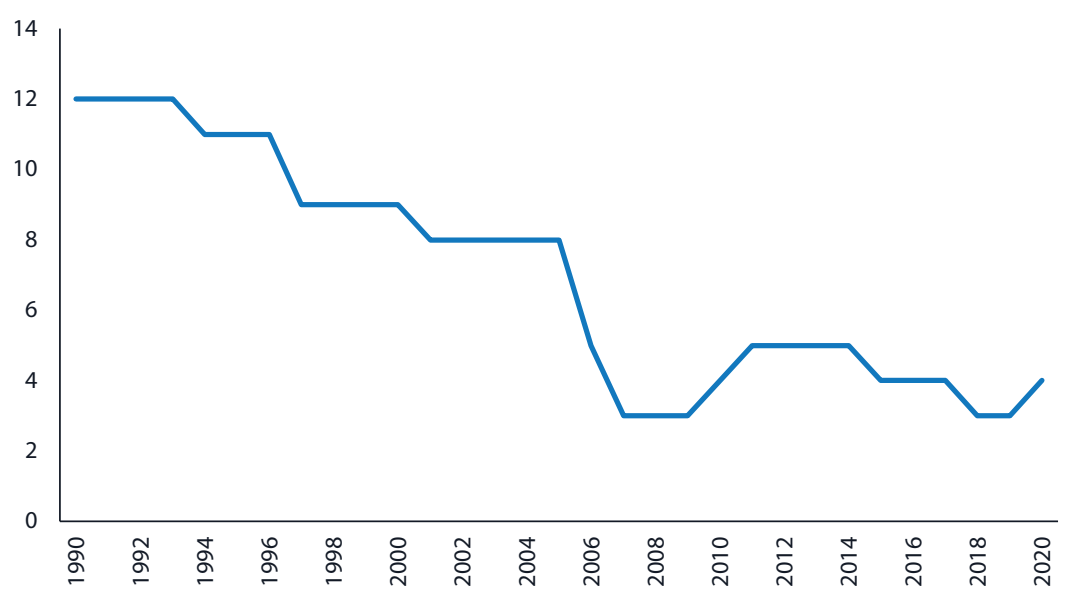

Fuente: Elaboración propia en base a OCDE $(2018,2020)$.

Nota: El número de países de la OCDE que cuenta con un impuesto al patrimonio aumentó de tres a cuatro tras la incorporación de Colombia a este organismo en abril de 2020.

La eliminación del impuesto al patrimonio en países de la OCDE se ha justificado tanto en su baja recaudación fiscal como en sus efectos adversos sobre la actividad económica. A modo de ejemplo, cuando Suecia eliminó el impuesto al patrimonio en 2007, el ministro de Hacienda de la época, Anders Borg, justificó su eliminación como una "política de impulso a la actividad empresarial para dinamizar el empleo" y aseguró que la medida tendría "un impacto real sobre la disposición a invertir en el país.13 En el caso de Francia, al momento de eliminar el impuesto al patrimonio en 2017, el primer ministro Edouard Philippe afirmó que "al menos 10.000 contribuyentes de altos ingresos habían trasladado capitales fuera de Francia debido al impuesto al patrimonio".14 Más allá de esta evidencia anecdótica, a continuación se analizan los principales efectos macroeconómicos del impuesto al patrimonio de acuerdo con la teoría

\footnotetext{
${ }^{13}$ Ver declaraciones del exministro de Hacienda de Suecia, Anders Borg (septiembre, 2017). Disponible en: www.ft.com/content/d6f77584-dd4a-11db-8d42-000b5df10621 [20 de octubre 2020].

${ }^{14}$ Ver declaraciones del exprimer ministro de Francia, Edouard Philippe (septiembre, 2017). Disponible en: www.bloomberg.com/news/articles/2017-09-28/france-s-wealth-tax-hasdriven-10-000-people-abroad-pm-says [20 de octubre 2020].
} 
económica y la evidencia empírica. En primer lugar, se analizan los efectos macroeconómicos que se desprenden de la teoría económica. Posteriormente, se documenta el impacto económico que tuvo el impuesto al patrimonio en diversos países de la OCDE que lo han implementado. Finalmente, se discute la magnitud de estos efectos macroeconómicos, utilizando las elasticidades del stock de capital estimadas por la literatura económica.

\section{Principales efectos macroeconómicos}

En un mundo altamente globalizado, un impuesto al patrimonio puede generar un éxodo de capitales que repercute sobre la inversión y la capacidad productiva de la economía. La movilidad del capital en respuesta a incentivos tributarios o en respuesta a un contexto de incertidumbre política respecto de medidas tributarias en el futuro, ha sido ampliamente documentada en la literatura económica. Le y Zak (2006) muestran que los flujos de capital en países en desarrollo presentan una alta sensibilidad a los riesgos políticos asociados a medidas tributarias, mientras que Alam y Quazi (2003) documentan que los cambios tributarios tienen un efecto significativo sobre las salidas de capitales. Por otro lado, estudios recientes de Moretti y Wilson $(2017,2019)$ muestran que la ubicación geográfica de los contribuyentes de altos ingresos en Estados Unidos es altamente sensible a las condiciones tributarias. En el caso de Francia, que se analizará con mayor detalle en la próxima sección, las salidas de capital se estimaron en más de 10.000 millones de euros por año $(0,5 \%$ del PIB), lo que podría haber reducido la recaudación fiscal en un monto superior a lo recaudado por el impuesto al patrimonio.

Un segundo efecto macroeconómico se refiere a una disminución en los incentivos al ahorro y la inversión. Mirrlees et al. (2011) señalan que un impuesto al patrimonio es, en la práctica, un impuesto sobre el retorno del capital, lo que distorsiona las decisiones de consumo y ahorro de las personas, y disminuye los incentivos a la inversión. Fama (2020) indica que el impuesto al patrimonio puede aumentar significativamente el costo del capital, lo que implica que diversos proyectos de inversión dejen de ser rentables, y que un impuesto al patrimonio relativamente pequeño implica un impuesto relativamente alto sobre los flujos de ingreso generados por los activos gravados. En esa línea, el ejemplo de- 
sarrollado por Cordero y Vergara (2020) es ilustrativo: considerando los retornos del capital en la actualidad, una tasa de impuesto de $1 \%$ sobre el stock de capital puede traducirse en una tasa de $50 \%$ sobre el retorno del capital. Además, se debe considerar que el stock de capital ya fue gravado a través del flujo que lo generó, el cual estuvo sujeto a impuestos sobre el ingreso personal y al impuesto corporativo, entre otros.

Un tercer efecto macroeconómico se refiere a que el impuesto al patrimonio puede disminuir el precio de los activos gravados. Lo anterior no solo afectaría a los contribuyentes de alto patrimonio, sino también a todos los hogares y empresas que cuenten con alguno de estos activos. En particular, Fama (2020) argumenta que si los precios de los activos reflejan los retornos netos de impuestos esperados por sus inversionistas, el impuesto al patrimonio puede disminuir significativamente el precio de tales activos. A modo de ejemplo, Fama considera un caso extremo en que, para un activo en particular, todo el efecto del impuesto al patrimonio se materializa a través de su precio. En tal escenario, y considerando una tasa de retorno de $5 \%$ sobre el capital, cada punto de impuesto al patrimonio disminuiría en $17 \%$ el precio del activo. ${ }^{15}$ En la práctica, la magnitud de este efecto dependerá de las características propias de cada activo gravado. Sin embargo, el ejemplo de Fama permite ilustrar que una tasa de impuesto al patrimonio relativamente baja puede afectar significativamente el precio de algunos activos.

Otro efecto se refiere a los costos de eficiencia para el Estado. El impuesto al patrimonio implica destinar recursos fiscales a la avaluación y tasación de diversos activos que conforman el patrimonio del contribuyente. En el caso de Chile, esto es especialmente cierto para los activos no financieros, que no son reportados a las entidades fiscalizadoras. Como argumentan Scheuer y Slemrod (2020), un criterio de avaluación heterogéneo de los activos podría provocar inequidad horizontal y una fuga hacia activos que pueden ser más fácilmente subvalorados. Por otro lado, el impuesto al patrimonio aumenta los incentivos a la evasión y la elusión, lo que puede afectar la recaudación obtenida por otros tipos de impuestos (OCDE 2018).

\footnotetext{
${ }^{15}$ Considerando el ejemplo de Fama (2020) para el caso en que el ajuste ocurre completamente a través del precio del activo, el nuevo precio tras el impuesto a la riqueza corresponde a $P^{\prime}=\frac{r P_{0}}{r+t}$, donde $P_{0}$ es el precio del activo antes del impuesto a la riqueza, $r$ es la tasa de retorno del activo y $t$ es la tasa de impuesto a la riqueza.
} 
Finalmente, un elemento a favor del impuesto al patrimonio, en relación con los impuestos que se aplican directamente sobre las rentas del capital, es que mientras estos últimos castigan el éxito empresarial, el impuesto al patrimonio establece una tasa homogénea sobre los contribuyentes de mayor riqueza. En particular, un empresario que realiza inversiones más rentables terminará pagando una mayor tasa de impuestos bajo un esquema que grava las rentas del capital, lo que no ocurre bajo el impuesto al patrimonio tradicional. Como muestran Guvenen et al. (2019), este efecto genera que la carga tributaria se redistribuya desde los empresarios más productivos hacia los menos productivos, lo que terminaría por aumentar la productividad agregada de la economía. Sin embargo, y como se analizará en la siguiente sección (3), la recaudación fiscal del impuesto al patrimonio ha sido particularmente baja en los países que lo han implementado, por lo que difícilmente podría sustituir la recaudación obtenida a través de otros tipos de impuestos.

\section{Evidencia empírica en países de la OCDE}

Históricamente, la evidencia empírica en torno a los efectos económicos del impuesto al patrimonio ha sido escasa, lo cual está vinculado a las restricciones en el acceso a datos sobre la riqueza de los contribuyentes y a las dificultades metodológicas para identificar un efecto causal. En lo más reciente, la propuesta de un impuesto global a la riqueza promovida por Piketty (2014), las mejoras en el acceso a datos administrativos y el avance de nuevas metodologías econométricas han propiciado el surgimiento de estudios empíricos en torno al impuesto al patrimonio. A continuación, se revisan un conjunto de trabajos que abordan los efectos del impuesto al patrimonio en la experiencia de diversos países de la OCDE (ver Tabla 1).

\section{Suiza}

Suiza es uno de los pocos países de la OCDE que mantiene un impuesto al patrimonio en la actualidad. En particular, Suiza establece una tasa de impuesto a la riqueza personal neta que varía entre $0,05 \%$ y 0,5\%, a nivel de provincias y municipios. Es precisamente esta variabilidad la que explotan Brülhart et al. (2020) para analizar el efecto del impuesto al patrimonio 
Tabla 1. EFECTOS ECONÓMICOS DEL IMPUESTO AL PATRIMONIO EN PAÍSES DE LA OCDE

\begin{tabular}{|c|c|c|c|}
\hline País & Estudio & Variable de interés & Efectos económicos \\
\hline Suiza & Brülhart et al. (2020) & $\begin{array}{l}\text { Fuga de capitales } \\
\text { Precio de activos } \\
\text { Ahorro de hogares } \\
\text { Evasión y elusión } \\
\text { tributaria }\end{array}$ & $\begin{array}{l}\text { - Disminución de } 1 \text { punto porcentual } \\
\text { (pp) en el impuesto al patrimonio } \\
\text { aumenta } 43 \% \text { la riqueza reportada por } \\
\text { los hogares. } \\
\text { - Un } 24 \% \text { del efecto se explica por } \\
\text { migración de contribuyentes, un } 20 \% \\
\text { por aumento en el precio de activos } \\
\text { inmobiliarios y un } 6 \% \text { por mayor ahorro } \\
\text { de los hogares. El resto del efecto res- } \\
\text { pondería a evasión y elusión tributaria, } \\
\text { entre otros factores. }\end{array}$ \\
\hline Dinamarca & Jakobsen et al. (2020) & $\begin{array}{l}\text { Riqueza reportada } \\
\text { Stock de capital }\end{array}$ & $\begin{array}{l}\text { - Disminución de } 1 \text { pp en el impuesto al } \\
\text { patrimonio aumenta entre } 17 \% \text { y } 25 \% \\
\text { la riqueza reportada por los hogares en } \\
\text { un plazo de } 8 \text { años. } \\
\text { - Elasticidad de largo plazo del stock de } \\
\text { capital se estima entre } 0,77 \text { y } 1,15 \text {. }\end{array}$ \\
\hline Francia & Pichet (2007) & Fuga de capitales & $\begin{array}{l}\text { - Fuga de capitales se estima en más } \\
\text { de } \$ 10.000 \text { millones de euros por año } \\
(0,5 \% \text { del PIB). } \\
\text { - El efecto acumulado de las salidas de } \\
\text { capital reduce la recaudación fiscal en } \\
\text { hasta } 7.000 \text { millones de euros por año } \\
(0,35 \% \text { del PIB). }\end{array}$ \\
\hline
\end{tabular}

Holanda Zoutman (2018) Riqueza reportada - Reforma de 2001 aumentó el impuesto al patrimonio, disminuyendo la riqueza reportada por los hogares en $14 \%$ por cada punto de impuesto.

España Durán-Cabré et al. Riqueza reportada • Reintroducción del impuesto al patri(2019) monio en 2011 disminuyó en 32\% la riqueza reportada por los hogares por cada punto de impuesto.

Suecia Seim (2017)

Riqueza reportada • Un aumento de 1 punto porcentual en el impuesto al patrimonio reduce la riqueza reportada por los hogares en $0,3 \%$.

Colombia Londoño-Velez y Ávi- Riqueza reportada • Un aumento de $1 \%$ en el impuesto al la-Mahecha (2018) patrimonio se traduce en una disminución de $2 \%$ en la riqueza reportada por los hogares.

Fuente: Elaboración propia en base a los estudios citados. 
sobre la riqueza reportada por los hogares, ${ }^{16}$ así como para descomponer este efecto en distintos mecanismos económicos. Los resultados del estudio son elocuentes: una disminución de 1 punto porcentual en el impuesto al patrimonio aumenta en $43 \%$ la riqueza reportada por los hogares.

Al analizar los mecanismos que impulsan el aumento de la riqueza reportada por los hogares en Suiza, se encuentra que un $24 \%$ del efecto se explica por la movilidad de los contribuyentes. Así, los contribuyentes se desplazan hacia las áreas del país con menores impuestos al patrimonio, lo que implica una salida de capitales desde las áreas con mayores tasas de impuestos. Por otro lado, un $20 \%$ del efecto se explica por un aumento en el precio de los activos inmobiliarios como consecuencia de un menor impuesto al patrimonio. Así, los costos económicos asociados a implementar un impuesto al patrimonio podrían ser asumidos no solo por los contribuyentes que pagan el impuesto, sino por todos los hogares que ven afectado el precio de sus activos inmobiliarios. Sin embargo, cabe señalar que los efectos estimados para el caso de Suiza consideran el valor reportado de los activos, el que no necesariamente equivale a su valor efectivo.

Brülhart et al. (2020) también encuentran que un $6 \%$ del aumento de la riqueza reportada se debe a un aumento en la tasa de ahorro de los hogares ante un menor impuesto a la riqueza. Si bien la magnitud de este efecto es más acotada que en el caso de los mecanismos anteriores, las decisiones de consumo y ahorro de los hogares tienen un efecto acumulativo a través del tiempo, lo que puede tener un impacto significativo sobre el stock de capital en el largo plazo, afectando la productividad y el empleo. El resto del efecto sobre la riqueza reportada por los hogares se atribuye, entre otros factores, a estrategias de evasión y elusión tributaria. Es decir, una reducción del impuesto al patrimonio permitiría reasignar recursos desde un uso claramente ineficiente, como son las estrategias de evasión y elusión tributaria, hacia actividades más productivas.

\section{Dinamarca}

Jakobsen et al. (2020) analizan el efecto del impuesto al patrimonio sobre las decisiones de consumo y ahorro de los contribuyentes afectados

\footnotetext{
${ }^{16}$ La riqueza reportada por los hogares corresponde al nivel de riqueza registrada en los formularios oficiales para el pago de impuestos, de acuerdo con los activos que se consideran imponibles en cada país.
} 
por el impuesto. En particular, se estima el efecto de la reforma de 1989, la cual redujo la tasa de impuesto a la riqueza desde $2,2 \%$ a $1 \%$, sobre la acumulación de riqueza de los hogares. Luego, se incorporan estos resultados en un modelo teórico que permite estimar las elasticidades de largo plazo del stock de capital respecto de la tasa de retorno del capital después de impuestos. Al igual que en el caso de Suiza, se encuentra que el impuesto al patrimonio afecta significativamente la riqueza reportada por los hogares. En particular, una reducción de 1 punto porcentual en el impuesto a la riqueza aumenta la riqueza reportada por los hogares afectados por el impuesto entre $17 \%$ y $25 \%$, en un rango de ocho años. Dado que los autores utilizan registros administrativos para el pago de impuestos, no es posible identificar qué proporción de este efecto se explica por un aumento efectivo de la riqueza y qué parte representa un subreporte previo de la riqueza en respuesta al impuesto.

Al incorporar estos resultados empíricos en un 'modelo del ciclo de vida' extendido, los autores estiman que la elasticidad de largo plazo del stock de capital, con respecto a la tasa de retorno después de impuestos, se ubica en un rango entre 0,77 y 1,15 para los contribuyentes afectados por el impuesto. Esto quiere decir que si la tasa de retorno del capital disminuye un $10 \%$, el stock de capital de largo plazo disminuye entre $7,7 \%$ y $11,5 \%$. Cabe señalar que este cálculo se refiere a la proporción del stock de capital que está en manos de los contribuyentes afectados por el impuesto. Por su parte, el efecto sobre el stock de capital agregado dependerá de la distribución del stock de capital en la población, es decir, de qué porcentaje del stock de capital está concentrado en los contribuyentes de alto patrimonio. Este punto se analiza con mayor detalle en la sección subtitulada 'Orden de magnitud'.

\section{Francia}

El caso de los impuestos a la riqueza en Francia ha tenido una alta repercusión mediática en los años recientes. Lo anterior se debe a la implementación del llamado 'impuesto a los súper-ricos' por parte del presidente François Hollande en 2012, que solo duró dos años en ejercicio, y a la abolición del histórico impuesto al patrimonio por parte del presidente Emmanuel Macron en 2017. Si bien no se han desarrollado estudios empíricos recientes que aborden, de manera rigurosa, el efecto de tales 
impuestos sobre la economía, cabe al menos analizar la magnitud de las salidas de capital que motivaron su eliminación. ${ }^{17}$

Pichet (2007) estima que las salidas de capital desde que se reinstauró el impuesto a la riqueza personal neta en 1988 hasta 2007 alcanzaron los 200.000 millones de euros. Esta cifra implica que, en promedio, cada año las salidas de capital fueron superiores a 10.000 millones de euros (en torno a $0,5 \%$ del PIB). Si bien es difícil obtener una estimación precisa de cuánto hubiesen disminuido las salidas de capital de no haberse implementado el impuesto a la riqueza, el estudio sugiere que el valor presente neto del impuesto puede haber sido negativo. En particular, el estudio estima que el efecto acumulado de las salidas de capital se traduce en una disminución de la recaudación fiscal de hasta 7.000 millones de euros por año $(0,35 \%$ del PIB), lo que es muy superior a la recaudación anual del impuesto a la riqueza, que alcanzaba los 4.400 millones de euros en ese entonces (0,23\% del PIB).

\section{Holanda, España, Suecia y Colombia}

El principal foco de la literatura reciente ha estado en estimar los efectos del impuesto al patrimonio sobre la riqueza reportada por los hogares. Si la riqueza de los hogares tiene una alta sensibilidad al impuesto al patrimonio, su implementación se traducirá en una disminución del stock de capital, con sus consecuentes efectos sobre el crecimiento, la productividad y el empleo. En esa línea, se han realizado estudios recientes para los casos de Holanda, España, Suecia y Colombia.

Zoutman (2018) analiza el caso holandés utilizando la reforma implementada en 2001, en la cual se aumentó la tasa del impuesto al patrimonio desde $0,8 \%$ a 1,2\%, además de disminuir el umbral de riqueza desde el cual se cobraba el impuesto. En línea con lo observado en los casos de Suiza y Dinamarca, el estudio encuentra una caída significativa de la riqueza reportada como consecuencia del aumento del impuesto. En particular, se estima que un punto adicional de impuesto al patrimonio disminuiría en $14 \%$ la riqueza reportada por los hogares en un plazo de cuatro años, lo cual incorpora tanto disminuciones efectivas

\footnotetext{
$\overline{17}$ Ver declaraciones del primer ministro de Francia, Edouard Philippe (septiembre, 2017). Disponibles en: www.bloomberg.com/news/articles/2017-09-28/france-s-wealth-tax-hasdriven-10-000-people-abroad-pm-says [20 de octubre 2020].
} 
en la riqueza como posibles estrategias de evasión y elusión. Zoutman (2018) sugiere que, debido a que las elasticidades de corto y largo plazo son similares, y a que reducir la riqueza efectiva puede tomar tiempo, este resultado respondería más a un subreporte de la riqueza que a una disminución efectiva de la riqueza. Sin embargo, a partir de los datos disponibles no es posible analizar empíricamente la magnitud relativa de ambos efectos.

Durán-Cabré et al. (2019) analizan el caso de España tras la reintroducción del impuesto al patrimonio en 2011, particularmente para los contribuyentes de Cataluña. En línea con los estudios analizados, se encuentra una caída significativa de la riqueza reportada por los hogares tras la implementación del impuesto, la cual disminuye 32\% por un punto de impuesto al patrimonio en un horizonte de cuatro años. Este efecto se explica principalmente por medidas para eludir el pago de impuestos a través de cambios en la composición del portafolio de activos. Lo anterior conlleva consecuencias sobre la eficiencia de la asignación de recursos en la economía, ya que se opta por inversiones potencialmente menos productivas en la medida en que faciliten el subreporte de la riqueza personal.

Seim (2017) encuentra un efecto en la misma dirección para el caso de Suecia, pero con un orden de magnitud significativamente menor (una reducción de 0,3\% de la riqueza reportada por los hogares por cada punto de impuesto al patrimonio). Como argumentan Jakobsen et al. (2020), lo anterior respondería a la estrategia empírica utilizada en el estudio. En particular, Seim (2017) utiliza una estrategia de bunching, la que permite identificar efectos causales a través del análisis de contribuyentes cerca del umbral de riqueza en que se comienza a cobrar el impuesto. Esta estrategia es efectiva para identificar efectos de evasión y elusión tributaria, pero no incorpora adecuadamente los cambios en la riqueza efectiva de los contribuyentes.

Utilizando una estrategia empírica similar a Seim (2017), LondoñoVelez y Ávila-Mahecha (2018) encuentran un efecto de mayor magnitud para el caso de Colombia, lo que se asocia con mayores dificultades para hacer cumplir este impuesto en países en desarrollo. En particular, el estudio estima que un aumento de $1 \%$ en el impuesto al patrimonio se traduce en una disminución de $2 \%$ en la riqueza reportada por los hogares en el corto plazo. Este trabajo muestra que existe una clara respuesta de los agentes económicos ante un impuesto al patrimonio, dado que los 
contribuyentes inmediatamente realizan un subreporte de sus activos para ubicarse por debajo del umbral de este impuesto. Por otro lado, se muestra que es posible disminuir las prácticas de evasión y elusión mediante políticas que promuevan el reporte de activos, como un esquema voluntario de repatriación de activos. Sin embargo, es difícil estimar la magnitud relativa de este efecto, dado que no se cuenta con información sobre los activos que poseen los contribuyentes locales en el exterior, especialmente en el caso de que estén ubicados en paraísos fiscales.

\section{Orden de magnitud}

En qué medida un impuesto al patrimonio pueda traducirse en efectos significativos sobre la economía está estrechamente relacionado con la elasticidad del stock de capital de los contribuyentes afectados por el impuesto. Esta elasticidad se define como el cambio porcentual en el stock de capital que ocurre frente a un pequeño cambio en la tasa de retorno del capital. En un estudio reciente, Saez y Stantcheva (2018) muestran formalmente cómo la elasticidad de largo plazo del stock de capital determina la magnitud de los efectos de un impuesto al patrimonio sobre la actividad económica. Para entender esta relación, se debe considerar primero que el impuesto al patrimonio genera una disminución en la tasa de retorno del capital. Siguiendo la formalización de Jakobsen et al. (2020), el cambio porcentual en la tasa de retorno del capital $(\Delta \% r)$ está dado por la ecuación (1), donde ' $t$ ' representa la tasa de impuesto al patrimonio y ' $r$ ' corresponde a la tasa de retorno del capital previo a establecer este impuesto.

$$
\Delta \% r=\frac{-\Delta \% t(1+r)}{(1-t)(1+r)-1}
$$

La disminución en el retorno del capital inducida por un impuesto al patrimonio se traduce en un menor stock de capital, mientras que el orden de magnitud de este efecto está dado por la elasticidad del stock de capital. Lo anterior también dependerá de qué porcentaje del stock de capital está en manos de los contribuyentes afectados por el impuesto. Si esta elasticidad es alta, y un porcentaje relevante del capital está concentrado en los contribuyentes de mayor riqueza, incluso un impuesto al patrimonio aparentemente pequeño puede tener implicancias significativas sobre el stock de capital y, por tanto, sobre el producto de largo plazo. 
El valor de la elasticidad de largo plazo del stock de capital es una pregunta netamente empírica. La mayoría de los estudios relacionados al impuesto al patrimonio que se han analizado estiman efectos de corto plazo (Brülhart et al. 2020; Durán-Cabré et al. 2019; Londoño-Velez y Ávila-Mahecha 2018; Seim 2017; Zoutman 2018), los que se acotan a un horizonte menor o igual a cinco años. Sin embargo, utilizando el caso de Dinamarca, Jakobsen et al. (2020) proporciona una estimación de la elasticidad de largo plazo del stock de capital. En particular, se incorporan las estimaciones empíricas en un modelo de 'ciclo de la vida' que permite computar las elasticidades de largo plazo. De acuerdo con estas estimaciones, la elasticidad de largo plazo del stock de capital se ubicaría en un rango de 0,77 a 1,15. A modo de ejemplo, y asumiendo una tasa de retorno del capital de 5\% (en línea con lo utilizado por Jakobsen et al. 2020), un impuesto al patrimonio de $1 \%$ reduce la tasa de retorno del capital en $27 \%$ (ver Ecuación 1). Luego, considerando el rango de elasticidades estimado, el stock de capital de los contribuyentes afectados por el impuesto al patrimonio se reduciría entre $21 \%$ y $31 \%$.

Para estimar cuál sería el efecto sobre el stock de capital agregado $y$, por tanto, sobre el producto de largo plazo, es necesario conocer qué porcentaje de la riqueza está en manos de los contribuyentes afectados por el impuesto al patrimonio. Si bien este cálculo es difícil de realizar en términos técnicos, Saez y Zucman (2019b) estiman que, en Estados Unidos, al menos un $10 \%$ del stock de capital está en manos de los contribuyentes de más alto patrimonio (mayores a US\$50 millones). De esta forma, las elasticidades estimadas por la literatura económica sugieren que los efectos de un impuesto al patrimonio sobre la actividad económica son relevantes. En esta línea, Cournède, Fournier y Hoeller (2018) encuentran que aumentar (o implementar) impuestos al patrimonio está asociado con una caída significativa del producto en el largo plazo. Por otro lado, Hansson (2010) encuentra un efecto negativo de los impuestos al patrimonio sobre el crecimiento económico.

\section{Efectos redistributivos}

Una de las principales motivaciones para establecer un impuesto al patrimonio se refiere a mejorar la distribución del ingreso en la población. En ese sentido, Halvorsen y Thoresen (2019) muestran que el impuesto al 
patrimonio ha tenido un efecto positivo sobre la distribución del ingreso en el caso de Noruega, mientras que Guvenen et al. (2019) estiman un modelo parametrizado con datos de Estados Unidos y encuentran que el impuesto al patrimonio reduce ligeramente la desigualdad en el consumo. Si bien estos efectos redistributivos existen, la literatura sugiere que ellos son acotados y que existen otros mecanismos mejores que un impuesto al patrimonio para disminuir los niveles de desigualdad.

En primer lugar, y como muestran Cournède, Fournier y Hoeller (2018), los efectos redistributivos del impuesto al patrimonio son de baja magnitud y conllevan un costo social relevante. Más precisamente, el impuesto al patrimonio no solo reduce el producto en el largo plazo, sino que lo hace de manera transversal en diversos grupos de la población. De esta forma, incluso si la distribución del ingreso mejorara en la práctica, la caída en el nivel de actividad económica se traduce en menores ingresos para los sectores más vulnerables.

Gráfico 4. EFECTO DE TRANSFERENCIAS SOCIALES E IMPUESTOS SOBRE EL ÍNDICE DE GINI

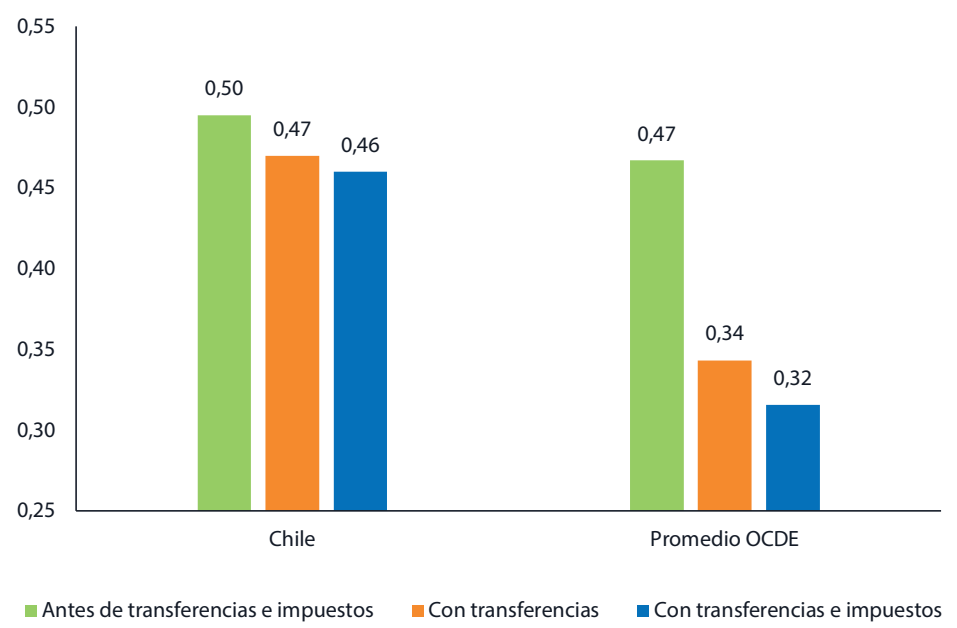

Fuente: Elaboración propia en base a OCDE (2020).

Por otro lado, si bien la política tributaria puede contribuir a mejorar la distribución del ingreso, la principal herramienta fiscal para reducir la desigualdad son las transferencias del gobierno. Como se observa en el Gráfico 4, en promedio los países de la OCDE reducen su Índice de Gini 
en 0,13 puntos gracias a las transferencias del gobierno y solo en 0,02 puntos adicionales mediante impuestos. Este punto es relevante para el caso de Chile, ya que el Índice de Gini se reduce en solo 0,03 puntos como consecuencia de las transferencias del gobierno. De esta forma, el espacio para reducir la desigualdad en Chile a través de transferencias sociales es significativamente mayor que hacerlo mediante el sistema tributario.

\section{Impacto fiscal del impuesto al patrimonio}

El impuesto al patrimonio ha sido un mecanismo de recaudación poco efectivo en los países de la OCDE. En 2017, la recaudación promedio entre los países de la OCDE que contaban con este tipo de impuesto fue de solo $0,16 \%$ del PIB (ver Gráfico 5). Al considerar la serie histórica desde 1990 a 2017, la recaudación promedio por impuesto al patrimonio en países de la OCDE es de 0,17\% del PIB y nunca fue superior a 0,2\% del PIB. En la misma línea, su contribución a la recaudación tributaria total ha sido poco significativa. En 2017, el impuesto al patrimonio aportó en promedio un 0,51\% del ingreso fiscal de los países de la OCDE que lo utilizaban, mientras que históricamente su aporte nunca ha sido superior a un 0,65\% del ingreso fiscal de los países OCDE.

Al desagregar a nivel de países, se observa que los miembros de la OCDE que eliminaron el impuesto al patrimonio recaudaban menos de $0,3 \%$ del PIB al momento de la derogación (ver Gráfico 6). ${ }^{18}$ En particular, Islandia recaudaba 0,3\% del PIB antes de eliminarlo en 2006, Francia un 0,22\% del PIB antes de eliminarlo en 2017, Suecia un 0,19\% del PIB antes de eliminarlo en 2007 y Holanda un 0,18\% del PIB antes de eliminarlo en 2001. Por su parte, Austria y Alemania recaudaban menos de 0,15\% del $\mathrm{PIB}$, mientras que Finlandia y Dinamarca ni siquiera alcanzaban un 0,1\% del PIB. Por otro lado, entre los países que mantienen vigente el impuesto, solo Suiza recauda más de $0,5 \%$ del PIB en la actualidad (1,08\% del PIB), mientras que Noruega recauda $0,45 \%$ del PIB y España apenas un $0,18 \%$ del PIB.

\footnotetext{
${ }^{18}$ El Gráfico 5 excluye el caso de Luxemburgo dado que, a pesar de haber implementado un impuesto a la riqueza personal hasta 2006 , no cuenta con cifras comparables en términos de recaudación.
} 
Gráfico 5. RECAUDACIÓN PROMEDIO DEL IMPUESTO A LA RIQUEZA EN PAÍSES OCDE

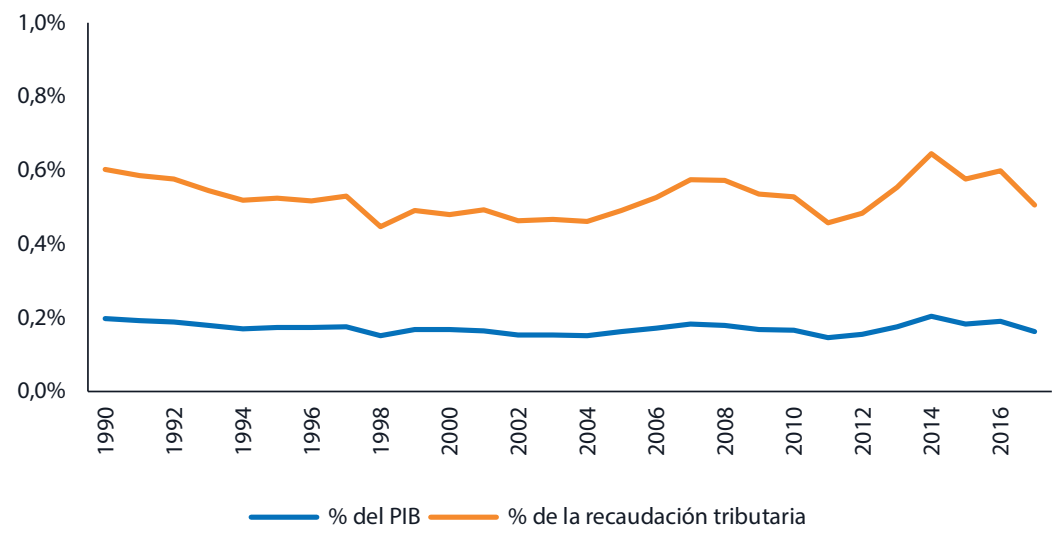

Fuente: Elaboración propia en base a OCDE (2020).

Gráfico 6. RECAUDACIÓN DEL IMPUESTO A LA RIQUEZA EN PAÍSES OCDE (\% PIB)

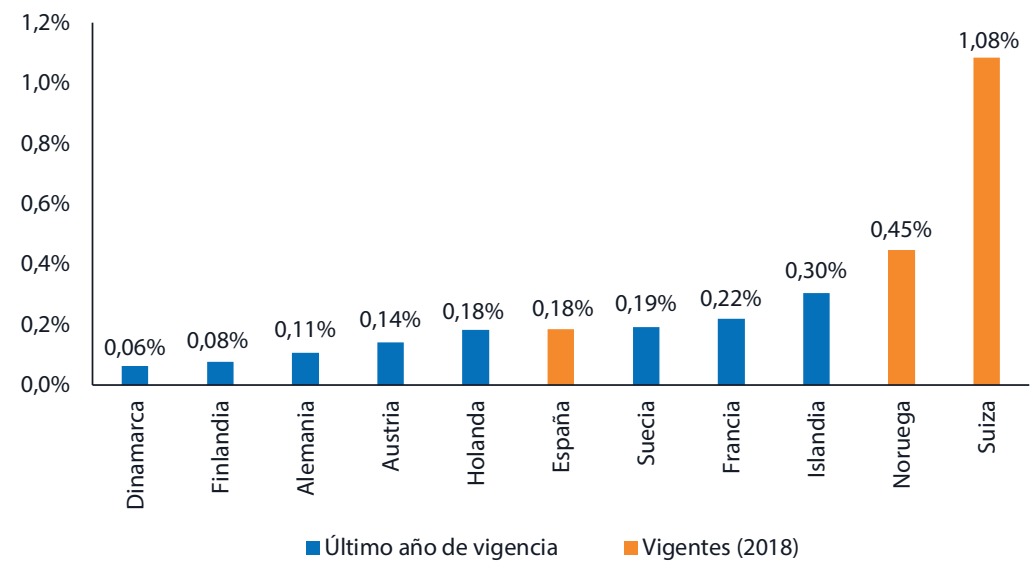

Fuente: Elaboración propia en base a OCDE (2020).

Las conclusiones respecto de la baja recaudación fiscal del impuesto al patrimonio son similares al considerar la máxima recaudación histórica de cada país. La mayoría de los países de la OCDE nunca logró una recaudación superior a 0,3\% del PIB, lo que sirve de referencia para cualquier estimación fiscal respecto de la implementación de un impuesto al patrimonio en Chile (OCDE 2020). Entre los países que lograron recaudar más de $0,3 \%$ del PIB, Suecia recaudó un máximo de 0,38\% del PIB en 1999, 
Islandia recaudó 0,5\% del PIB en 1993 y Noruega recaudó 0,53\% del PIB en 1995. Al igual que en el análisis anterior, se observa que Suiza es una excepción entre los países de la OCDE, ya que alcanzó una recaudación máxima de 1,09\% del PIB en 2017.

\section{Impuesto al patrimonio en Chile}

\section{Análisis de la propuesta}

El 26 de mayo de 2020, un grupo de parlamentarios presentó un Proyecto de Acuerdo que solicita la creación de un impuesto de 2,5\% sobre los patrimonios superiores a US\$10 millones. ${ }^{19}$ Posteriormente, el 1 de junio de 2020, se ingresó una Moción Parlamentaria que plantea una reforma constitucional para la creación de un impuesto de 2,5\% sobre los patrimonios superiores a US\$22 millones. ${ }^{20}$ En términos generales, el mecanismo propuesto es similar al impuesto al patrimonio discutido en la sección anterior para los países OCDE. Sin embargo, existen algunos aspectos técnicos de la propuesta presentada para Chile que es relevante analizar.

En primer lugar, el impuesto al patrimonio en los países OCDE se define como un impuesto sobre la riqueza personal neta de deudas (OCDE 2018), mientras que la Moción Parlamentaria sugiere un impuesto sobre el 'patrimonio bruto', lo que correspondería a los activos totales de una persona sin importar sus deudas. Si bien el proyecto no define con precisión cuáles activos financieros y no financieros se considerarán parte del patrimonio, llama la atención la utilización del concepto de 'patrimonio bruto', considerando que tanto en la experiencia internacional como en la literatura económica se ha definido el patrimonio como la riqueza personal neta de deudas.

Saez y Zucman (2019a, 4), dos de los principales impulsores del impuesto al patrimonio en la actualidad, señalan que "la medida estándar y ampliamente considerada de la riqueza de los hogares considera sus activos financieros y no financieros avaluados a su precio de mercado,

\footnotetext{
${ }^{19}$ Ver Proyecto de Resolución N ${ }^{\circ}$ 1088. Disponible en: https://www.camara.cl/verdoc.aspx? prmid $=6280 \&$ prmtipo $=$ PROYECTO_ACUERDO [20 de octubre 2020].

${ }^{20}$ Ver Moción Parlamentaria correspondiente al Boletín №13555-07. Disponible en: https:// www.camara.cl/verDoc.aspx?prmID=13876\&prmTIPO=INICIATIVA [20 de octubre 2020].
} 
neto de deudas". Así también lo consideran la OCDE (2018) y los reportes de organismos especializados, como la Worldwide Estate Inheritance Tax Guide (Ernst \& Young 2019). Por su parte, los países de la OCDE que mantienen impuestos al patrimonio en la actualidad (España, Noruega y Suiza) definen tales impuestos sobre la riqueza personal neta de deudas. En la misma línea, las propuestas elaboradas por algunos precandidatos demócratas en Estados Unidos (Elizabeth Warren y Bernie Sanders), en el contexto de las elecciones presidencias de 2020, consideraban un impuesto sobre la riqueza personal neta de deudas. ${ }^{21}$ Así, la utilización del concepto de patrimonio 'bruto' parece ser una imprecisión técnica de la Moción Parlamentaria, que se aleja tanto de su conceptualización económica como de la experiencia de otros países.

Un segundo punto a considerar es que, de acuerdo con la Moción Parlamentaria, el impuesto al patrimonio se cobraría por una sola vez, mientras que los impuestos al patrimonio implementados por países de la OCDE durante las últimas décadas se cobran de manera regular. Desde una perspectiva histórica, hay evidencia de impuestos al patrimonio por una sola vez en los años posteriores a las guerras mundiales. Esta medida generó problemas de implementación, fugas de capitales y una baja efectividad tributaria en la mayoría de los casos, mientras que se observan experiencias más positivas en los casos puntuales de Japón y Alemania tras la Segunda Guerra Mundial (Bach 2012; Bach, Beznoska y Steiner 2014). Uno de los estudios más reconocidos en esta área corresponde a Eichengreen (1989), el que plantea que el éxito de un impuesto al patrimonio por una sola vez radica en que sea percibido como excepcional por parte de los agentes económicos. En la misma línea, Advani, Chamberlain y Summers (2020) sugieren que un impuesto al patrimonio por una sola vez sería efectivo para el caso del Reino Unido, en la medida en que se cumplan dos condiciones principales: que los contribuyentes no ajusten su comportamiento en anticipación al impuesto y que sea creíble que el impuesto no se repetirá en el tiempo.

Si bien en teoría un impuesto al patrimonio podría tener un carácter transitorio, en la práctica existe el riesgo latente de que tal impuesto se prolongue en el tiempo, lo que será incorporado en las expectativas de

\footnotetext{
${ }^{21}$ Ver propuestas de los precandidatos presidenciales en Estados Unidos. Disponibles en: https://berniesanders.com/issues/tax-extreme-wealth/, https://elizabethwarren.com/plans/ ultra-millionaire-tax [20 de octubre 2020].
} 
los agentes económicos. En ese sentido, la experiencia reciente de España es ilustrativa. Luego de eliminar el impuesto al patrimonio en 2008, este se reinstauró de manera transitoria durante 2011 para hacer frente a las dificultades fiscales que atravesaba el país a raíz de la crisis del euro (OCDE 2018). A pesar del carácter inicial del impuesto, y de que su impacto fiscal en términos de recaudación es relativamente bajo $(0,18 \%$ del PIB en 2018), este impuesto se mantiene vigente hasta la fecha.

En el caso de Chile, diversos impuestos que se han establecido en un formato transitorio han debido mantenerse en el tiempo para hacer frente a necesidades fiscales adicionales. A modo de ejemplo, este fue el caso del impuesto específico a los combustibles para financiar la reconstrucción del terremoto de 1985 (Ley № 18.502), del alza del IVA para financiar el plan AUGE en 2003 (Ley $N^{\circ}$ 19.888) y del aumento del Impuesto de Primera Categoría para financiar la reconstrucción tras el terremoto de 2010 (Ley $N^{\circ}$ 20.455). Por otro lado, lo ocurrido con el retiro de fondos previsionales durante 2020 refuerza el punto anterior. Si bien esta medida fue anunciada como una política excepcional y por una sola vez, en la práctica se aprobó un segundo retiro a los pocos meses de haberse implementado el primero. De esta forma, más allá de lo que se establezca en el texto legal, la promesa de un impuesto al patrimonio por una sola vez parece poco creíble en el contexto actual.

Un tercer punto a considerar es que una tasa de impuesto al patrimonio de 2,5\%, como propone la Moción Parlamentaria, es superior a las tasas observadas en la mayoría de las experiencias recientes de países OCDE. La tasa de impuesto al patrimonio propuesta para Chile equivale a cinco veces la tasa máxima vigente en Suiza (rango entre 0,05\% y 0,5\%), a prácticamente tres veces la tasa vigente en Noruega $(0,85 \%)$ y es superior a la tasa que tenía Francia hasta 2017 (rango entre 0,5\% y 1,5\%). Por otro lado, esta tasa solo es comparable a la experiencia de España, en que la tasa máxima a nivel de gobierno central es 2,5\%, aunque esta varía en las distintas comunidades autónomas. ${ }^{22}$

Finalmente, otro problema de diseño que tiene la Moción Parlamentaria que crea un impuesto al patrimonio es que, tal como está planteada, generaría un aumento discreto del pago del impuesto pasado un

\footnotetext{
${ }^{22}$ Mientras la Comunidad Autónoma de Madrid está exenta de impuesto a la riqueza personal, la tasa máxima de impuesto a la riqueza personal se eleva hasta 3,75\% en Extremadura.
} 
umbral (US\$22 millones). Este diseño tributario genera fuertes incentivos al subreporte en los contribuyentes con patrimonios cercanos al punto de corte, profundizando los problemas relacionados a la avaluación de activos no financieros señalados previamente.

\section{Estimación fiscal de la propuesta}

De acuerdo a lo señalado en el Proyecto de Acuerdo (26 de mayo de 2020), con el impuesto al patrimonio se espera recaudar más de US\$6.000 millones. La Moción Parlamentaria (1 de junio de 2020) precisó esta cifra y estima que se recaudará en torno a US\$6.500 millones. Esta estimación equivale a recaudar 2,3\% del PIB por concepto de impuesto al patrimonio. ${ }^{23} \mathrm{El}$ Gráfico 7 presenta el cálculo fiscal de la Moción Parlamentaria en comparación con las experiencias de los países OCDE que han utilizado un impuesto al patrimonio, considerando el máximo monto recaudado por cada país entre 1990 y 2018. Se observa que la estimación fiscal realizada para Chile es más del doble que la mayor recaudación alcanzada por un país OCDE mediante este tipo de impuestos, la que corresponde al caso de Suiza en 2017 (1,09\% del PIB). Además, la estimación fiscal para Chile es más de cuatro veces la máxima recaudación obtenida por Noruega $(0,53 \%$ del PIB en 1995$)$ e Islandia $(0,5 \%$ del PIB en 1993), y es varias veces superior a la máxima recaudación obtenida por cualquiera de los demás países de la OCDE.

A continuación se considera la recaudación fiscal por cada punto de impuesto al patrimonio, lo que facilita la comparación entre la estimación fiscal realizada para Chile y las experiencias de los países de la OCDE. Además, se considera la recaudación del último año en que el impuesto estuvo vigente en cada país $O C D E$, en lugar de su máxima recaudación histórica, de manera de considerar un período más reciente. Dado que la Moción Parlamentaria propone un impuesto al patrimonio de $2,5 \%$, su estimación fiscal (US\$6.500 millones) equivale a recaudar $0,92 \%$ del PIB por cada punto de impuesto al patrimonio. La Tabla 2 presenta información sobre los países OCDE que han implementado un impuesto con una tasa homogénea para todos los contribuyentes de alto

\footnotetext{
${ }^{23}$ Este cálculo considera el PIB de 2019 a precios corrientes publicado por el Banco Central de Chile, convertido a dólares utilizando el tipo de cambio promedio de 2019, de acuerdo con la misma institución.
} 
patrimonio, lo que permite calcular de manera directa la recaudación fiscal por cada punto de impuesto al patrimonio. ${ }^{24}$ En particular, para cada país se computa la recaudación por punto de impuesto al patrimonio como porcentaje del PIB, en su último año de vigencia o en 2018, en caso de aún estar vigente.

Gráfico 7. RECAUDACIÓN FISCAL POR IMPUESTO AL PATRIMONIO (\% PIB)

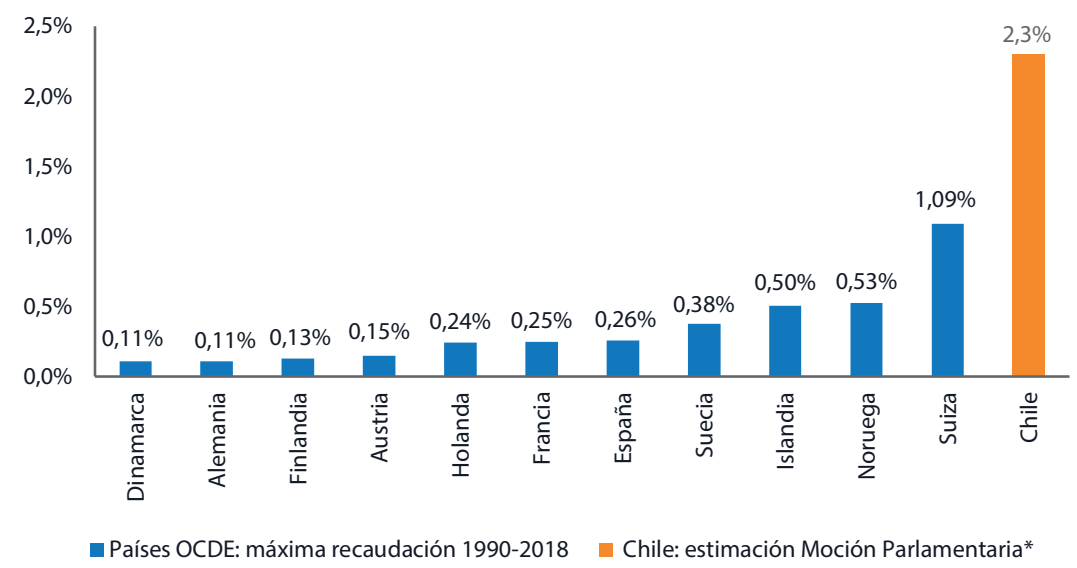

Fuente: Elaboración propia en base a $\operatorname{OCDE}(2018,2020)$ y Moción Parlamentaria (Boletín № 13555 07).

Tabla 2. PAÍSES OCDE QUE HAN IMPLEMENTADO UN IMPUESTO AL PATRIMONIO CON TASA HOMOGÉNEA

\begin{tabular}{lccc}
\hline País & $\begin{array}{c}\text { Último año de } \\
\text { vigencia }\end{array}$ & $\begin{array}{c}\text { Tasa de impuesto } \\
(\%)\end{array}$ & $\begin{array}{c}\text { Recaudación por punto } \\
\text { de impuesto (\% PIB) }\end{array}$ \\
\hline Austria & 1994 & 1,0 & 0,14 \\
Alemania & 1997 & 1,0 & 0,11 \\
Holanda & 2001 & 0,7 & 0,26 \\
Finlandia & 2006 & 0,8 & 0,10 \\
Islandia & 2006 & 1,5 & 0,20 \\
Suecia & 2007 & 1,5 & 0,13 \\
Noruega & Vigente (2018) & 0,85 & 0,53 \\
\hline
\end{tabular}

Fuente: Elaboración propia en base a OCDE $(2018,2020)$.

\footnotetext{
${ }^{24}$ Países como España, Suiza y Francia cuentan con tasas de impuesto a la riqueza progresivas de acuerdo con el monto del patrimonio, lo que dificulta el análisis anterior.
} 
El Gráfico 8 presenta los resultados de la Tabla 2 en comparación con la estimación que acompaña la Moción Parlamentaria de impuesto al patrimonio. Se observa que la recaudación estimada para Chile es muy superior a la experiencia previa de los países OCDE. En particular, la estimación realizada para Chile es más de ocho veces lo que recaudaban Alemania $(0,11 \%$ del PIB) y Finlandia $(0,1 \%$ del PIB) por cada punto de impuesto al patrimonio, en torno a siete veces lo que recaudaban Suecia $(0,13 \%$ del PIB) y Austria $(0,14 \%)$, más de cuatro veces lo que recaudaba Islandia (0,2\% del PIB), más de 3 veces lo que recaudaba Holanda $(0,26 \%$ del PIB) y un 75\% mayor que la recaudación que alcanza Noruega en la actualidad (0,53\% del PIB). ${ }^{25}$

Gráfico 8. RECAUDACIÓN FISCAL POR CADA PUNTO DE IMPUESTO AL PATRIMONIO (\% PIB)

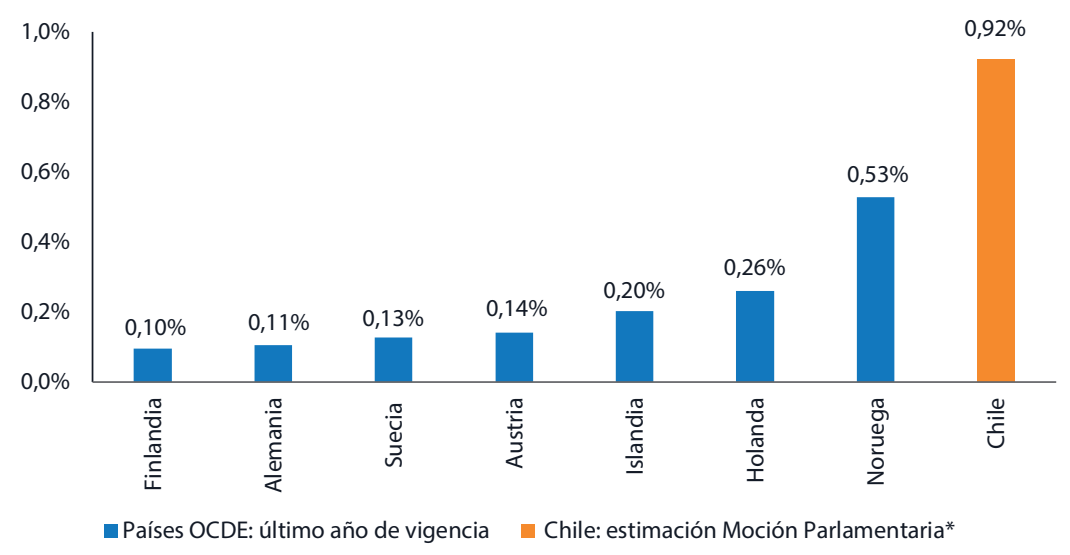

Fuente: Elaboración propia en base a $\operatorname{OCDE}(2018,2020)$ y Moción Parlamentaria (Boletín N 13555 07).

\footnotetext{
25 López y Sturla (2020) estiman que un impuesto al patrimonio con una tasa de 2,5\% tendría una recaudación de US\$4.923 millones, en caso de gravar a los patrimonios sobre US\$5 millones. Esta cifra también es inferior a lo estimado por la Moción Parlamentaria (US\$6.500 millones), a pesar de que esta última gravaría a un grupo más reducido de contribuyentes (patrimonios sobre US\$22 millones). Por otro lado, las estimaciones de López y Sturla (2020) utilizan como supuesto una tasa de evasión particularmente baja, siendo de solo 5\% para los 'súper-ricos'. Lo anterior contrasta con las estimaciones de Saez y Zucman (2019a) para Estados Unidos, quienes utilizan como supuesto una tasa de evasión de 15\% a 50\%, y sugieren que la tasa de evasión efectiva estaría en torno al 33\%.
} 


\section{Comparación entre Chile y la OCDE}

De acuerdo a la experiencia de los países OCDE, los cálculos fiscales que acompañan a la propuesta de impuesto al patrimonio para Chile están sobreestimados. Por otra parte, la evidencia empírica analizada en la sección 2, bajo el subtítulo 'Evidencia empírica en países de la OCDE', sugiere que el impuesto al patrimonio afecta el comportamiento de los agentes económicos, generando salidas de capital, disminuciones en el precio de los activos, menores tasas de ahorros, y mayores prácticas de elusión y evasión tributaria. En la práctica, el orden de magnitud de los efectos macroeconómicos está estrechamente relacionado con la elasticidad del stock de capital, cuyas estimaciones tienen, en general, un valor que no es despreciable en términos económicos.

Un punto a considerar es que, si bien la experiencia de los países de la OCDE provee un marco de referencia para entender los efectos que podría tener un impuesto al patrimonio, existen diferencias económicas relevantes entre Chile y algunos países de la OCDE. A modo de ejemplo, Suiza y Noruega mantienen impuestos al patrimonio en la actualidad, pero el PIB per cápita de estos países es más de 2,5 veces el de Chile (FMI 2020). ${ }^{26}$ Sin embargo, en términos del tamaño de la economía, las experiencias de Australia, Alemania y Dinamarca proveen una aproximación más cercana a la realidad de Chile. En particular, Alemania y Dinamarca mantuvieron su impuesto al patrimonio hasta 1997, año en que tenían un PIB per cápita de US\$26,259 y US\$29,784, respectivamente. Ambos ejemplos son significativamente más cercanos al contexto actual de Chile (US\$25,057 per cápita). Por su parte, Austria mantuvo su impuesto al patrimonio hasta 1994, año en que su PIB per cápita era inferior al de Chile en la actualidad, alcanzando los US\$23,660. De esta forma, si bien no es posible controlar por las múltiples diferencias económicas entre Chile y los demás países de la OCDE, algunos de los casos de eliminación de este impuesto ocurrieron cuando estos países tenían un nivel de ingreso similar al de Chile.

Un segundo punto que se debe considerar al comparar a Chile con los países de la OCDE se refiere a las diferencias en el diseño del impuesto al patrimonio. Como se analizó en la sección 'Análisis de la propuesta', el impuesto al patrimonio utilizado en los países de la OCDE se define so-

\footnotetext{
${ }^{26}$ Se considera el PIB per cápita en dólares a Paridad del Poder de Compra (PPP).
} 
bre el patrimonio neto de una persona, mientras que la propuesta para el caso de Chile hace referencia al patrimonio 'bruto'. Este concepto, que difiere de la conceptualización económica de riqueza personal, podría explicar parte de la diferencia entre la recaudación fiscal estimada en la Moción Parlamentaria y la experiencia de los países OCDE.

Otra diferencia abordada en dicha sección se refiere a que el proyecto de impuesto al patrimonio en Chile sería por una sola vez, mientras que los impuestos al patrimonio en los países de la OCDE se cobran de manera regular. Lo anterior podría explicar parcialmente una mayor recaudación fiscal. Sin embargo, en un contexto de alta movilidad del capital es difícil asegurar que los efectos documentados en las secciones anteriores, como son las salidas de capital y el comportamiento estratégico de los contribuyentes, no ocurrirán en la práctica. Ello es especialmente cierto al considerar que los agentes económicos incorporarán la posibilidad de que este impuesto se constituya en un impuesto permanente, tal como ocurrió en el caso de España.

Un elemento adicional se refiere a que Chile no tiene experiencia reciente en este tipo de impuestos, lo que podría traducirse en una menor efectividad en la recaudación respecto de los países OCDE analizados. Chile instauró un impuesto al patrimonio en 1968, pero este fue derogado hace más de cuatro décadas (1974). ${ }^{27}$ Si bien en la actualidad se cuenta con las contribuciones de bienes raíces y el impuesto a las herencias y donaciones, tales gravámenes no son equivalentes en complejidad a un impuesto sobre el patrimonio. En particular, se debe considerar la cantidad y diversidad de activos financieros y no financieros que se deberán avaluar, así como el número de contribuyentes que serán gravados en el mismo momento del tiempo.

Una comparación más precisa en términos de recaudación fiscal requeriría conocer mayores detalles técnicos de la propuesta realizada para Chile, especialmente en cuanto a los activos financieros y no financieros especíicos que serían gravados, así como los mecanismos de avaluación y recaudación que serían utilizados. En la práctica, existen factores que afectan la comparación entre Chile y la OCDE, cuya magnitud es difícil de estimar. Sin embargo, la diferencia entre la estimación de

\footnotetext{
${ }^{27}$ El gobierno del presidente Eduardo Frei Montalva creó un impuesto al patrimonio en 1968 (Ley $N^{\circ} 17.073$ ), el que posteriormente fue derogado por el Decreto de Ley 298 del año 1974.
} 
recaudación fiscal para Chile y lo observado en la experiencia de los países OCDE es demasiado grande como para hacer plausible la estimación de esta propuesta.

\section{Conclusiones}

En un contexto en que el país atraviesa su mayor recesión económica en casi cuatro décadas, se requiere un conjunto de medidas que promuevan la inversión nacional y extranjera, y que faciliten la creación de empleos. Por otro lado, es evidente que el país necesitará obtener recursos fiscales que permitan cubrir los gastos incurridos para enfrentar la crisis sanitaria y financiar las crecientes demandas sociales en materia previsional, educacional y de salud pública, entre otras. Para abordar ambos objetivos de manera conjunta, es esencial que las políticas tributarias utilizadas minimicen los efectos sobre el crecimiento económico y permitan una recaudación fiscal sustantiva a un bajo costo operacional. El impuesto al patrimonio falla en ambas dimensiones.

La evidencia presentada en este documento es elocuente en cuanto a los efectos del impuesto al patrimonio sobre la economía. El impuesto al patrimonio puede inducir salidas de capitales, reducir los incentivos al ahorro y la inversión, disminuir el precio de los activos gravados y generar altos costos de eficiencia para el Estado. El orden de magnitud de estos efectos dependerá de diversos factores, siendo la elasticidad del stock de capital uno de sus principales determinantes. La literatura económica sugiere que esta elasticidad tiene un valor que no es despreciable, lo que se traduce en una reducción del stock de capital y del producto de largo plazo. En términos de distribución del ingreso, se observa un efecto positivo por parte del impuesto al patrimonio, pero su alcance es limitado en relación con lo que es posible lograr mediante transferencias sociales. Por otro lado, la experiencia de los países de la OCDE que han utilizado este tipo de impuestos es ilustrativa respecto de su baja capacidad recaudatoria.

El análisis realizado en este artículo extiende un conjunto de trabajos elaborados en Clapes UC (González, Larraín y Perelló 2020a, 2020b). La motivación de estos trabajos proviene de la convicción de que la selección y el diseño de las políticas públicas es esencial para alcanzar los objetivos que nos planteamos como sociedad. En esta línea, hacemos un 
llamado de atención respecto de la forma en que se ha pretendido establecer un impuesto al patrimonio en Chile. En primer lugar, la utilización de mecanismos que están fuera del orden constitucional para la política tributaria establece un mal precedente para nuestra institucionalidad. Por otro lado, la propuesta de impuesto al patrimonio contiene diversas imprecisiones técnicas, efectos macroeconómicos adversos y una estimación fiscal alejada de la experiencia internacional. Así, evaluado por separado y en su conjunto, no cabe más que concluir que el impuesto al patrimonio es una política tributaria deficiente.

\section{Bibliografía}

Alam, I. y Quazi, R. 2003. Determinants of Capital Flight: An Econometric Case Study of Bangladesh. International Review of Applied Economics 17(1), 85-103. DOI: https://doi.org/10.1080/713673164.

Advani, A., Chamberlain, E. y Summers, O. 2020. A Wealth Tax for the UK. Wealth Tax Commission Final Report. London School of Economics and Political Science, Economic and Social Research Council, Warwick University. Disponible en: https://warwick.ac.uk/fac/soc/economics/research/centres/cage/news/awealth-tax-for-the-uk.pdf [17 de enero 2021].

Bach, S. 2012. Capital Levies: A Step towards Improving Public Finances in Europe. DIW Economic Bulletin 2(8), 3-11. Disponible en: https://www.diw.de/documents/ publikationen/73/diw_01.c.406665.de/diw_econ_bull_2012-08-1.pdf [17 de enero 2021].

Bach, S., Beznoska, M. y Steiner, V. 2014. A Wealth Tax on the Rich to Bring Down Public Debt? Revenue and Distributional Effects of a Capital Levy in Germany. Fiscal Studies 35(1), 67-89. DOI: https://doi.org/10.1111/j.1475-5890.2014.12023.x.

Banco Central de Chile 2020a. Base de datos estadísticos. Disponible en: https://si3. bcentral.cl/siete/ [1 de septiembre 2020].

Banco Central de Chile 2020b. Informe de política monetaria. Disponible en: https://www.bcentral.cl/contenido/-/detalle/informe-de-politica-monetariadiciembre-2020 [9 de diciembre 2020].

Brülhart, M., Gruber, J., Krapf, M. y Schmidheiny, K. 2020. Behavioral Responses to Wealth Taxes: Evidence from Switzerland. CESifo Working Paper Series. Disponible en: https://papers.ssrn.com/sol3/papers.cfm?abstract_id=3477721 [1 de septiembre 2020].

Centro de Encuestas y Estudios Longitudinales UC 2020. Encuesta de Empleo Covid-19 de julio. Disponible en: https://www.youtube.com/watch?v=3q9PMY3qehc\&t=8se [13 de agosto 2020].

Cordero, A. y Vergara, R. 2020. Algunas reflexiones sobre la propuesta de impuesto a la riqueza. Puntos de Referencia 524. Disponible en: https://www.cepchile.cl/cep/ puntos-de-referencia/puntos-de-referencia-2020/algunas-reflexiones-sobre-lapropuesta-de-impuesto-a-la-riqueza [17 de enero 2021].

Cournède, B., Fournier, J.M. y Hoeller, P. 2018. Public Finance Structure and Inclusive Growth. OECD 25. Disponible en: https://www.oecd-ilibrary.org/economics/ 
structure-des-finances-publiques-et-croissance-inclusive_42b878d8-fr [17 de enero 2021].

Durán-Cabré, J.M., Esteller-Moré, A. y Mas-Montserrat, M. 2019. Behavioural Responses to the (Re)Introduction of Wealth Taxes. Evidence from Spain. Working Paper 04. Institut d'Economia de Barcelona (IEB). Disponible en: https://papers.ssrn.com/ sol3/papers.cfm?abstract_id=3393016 [17 de enero 2021].

Eichengreen, B. 1989. The Capital Levy in Theory and Practice. National Bureau of Economic Research 3096. Disponible en: https://www.nber.org/papers/w3096 [17 de enero 2021].

Ernst \& Young 2019. Worldwide Estate Inheritance Tax Guide. Disponible en: https:// www.ey.com/en_gl/tax-guides/worldwide-estate-and-inheritance-taxguide-2020 [17 de enero 2021].

Fama, E.F. 2020. Wealth Taxes. Fama-Miller Working Paper. Disponible en: https:// papers.ssrn.com/sol3/papers.cfm?abstract_id=3403782 [17 de enero 2021].

Fuest, C., Neumeier, F., Stimmelmayr, M. y Stöhlker, D. 2018. The Economic Effects of a Wealth tax in Germany. ifo DICE Report 16(2), 22-26. Disponible en: https://www. econstor.eu/bitstream/10419/181277/1/dice-report-2018-2-50000000002757. pdf [17 de enero 2021].

González, H., Larraín, F. y Perelló, Ó. 2020a. Impuesto al patrimonio: Efectos macroeconómicos y fiscales. Documento de Trabajo Clapes UC 80. Disponible en: https://clapesuc.cl/investigacion/doc-trabajo-n80-impuesto-al-patrimonioefectos-macroeconomicos-y-fiscales [17 de enero 2021].

González, H., Larraín, F. y Perelló, Ó. 2020b. Impuesto al patrimonio II: Consideraciones institucionales y efectos económicos en países OCDE. Documento de Trabajo Clapes UC 83. Disponible en: https://clapesuc.cl/investigacion/doc-trabajono83-impuesto-al-patrimonio-ii-consideraciones-institucionales-y-efectoseconomicos-en-paises-ocde [17 de enero 2021].

Guvenen, F., Kambourov, G., Kuruscu, B., Ocampo-Diaz, S. y Chen, D. 2019. Use It or Lose It: Efficiency Gains from Wealth Taxation. National Bureau of Economic Research 26284. Disponible en: https://www.nber.org/papers/w26284 [17 de enero 2021].

Halvorsen, E. y Thoresen, T.O. 2019. Distributional Effects of a Wealth Tax under Lifetime-Dynastic Income Concepts. The Scandinavian Journal of Economics. DOI: https://doi.org/10.1111/sjoe.12392.

Hansson, A.. 2010. Is the Wealth Tax Harmful to Economic Growth? World Tax Journal 2(1), 19-34. Disponible en: https://www.sv.uio.no/econ/english/research/ centres/ofs/news-and-events/events/dokumenter/Hansson8march13.pdf [17 de enero 2021].

Honorable Cámara de Diputados de Chile 2020. Modifica la Carta Fundamental para establecer por única vez, un impuesto al patrimonio de determinadas personas naturales, destinado al financiamiento de una renta básica de emergencia. Boletín N 13555-07. Disponible en: https://www.senado.cl/appsenado/ templates/tramitacion/index.php?boletin_ini=13555-07 [17 de enero 2021].

Honorable Cámara de Diputados de Chile 2020. Proyecto de acuerdo de impuesto al patrimonio. Resolución 1088. Disponible en: https://www.camara.cl/verdoc.aspx ?prmid=6280\&prmtipo=PROYECTO_ACUERDO [17 de enero 2021].

FMI (Fondo Monetario Internacional) 2020. World Economic Outlook October. Disponible en: https://www.imf.org/en/Publications/WEO/Issues/2020/09/30/ world-economic-outlook-october-2020 [1 de octubre 2020]. 
INE (Instituto Nacional de Estadísticas) 2020. Boletín Empleo Nacional trimestre móvil mayo-junio-julio 2020. Disponible en: https://www.ine.cl/estadisticas/sociales/ mercado-laboral/ocupacion-y-desocupacion [1 de agosto 2020].

Jakobsen, K., Jakobsen, K., Kleven, H. y Zucman, G. 2020. Wealth Taxation and Wealth Accumulation: Theory and Evidence from Denmark. The Quarterly Journal of Economics 135(1), 329-388. DOI: https://doi.org/10.1093/qje/qjz032.

Le, Q.V. y Zak, P.J. 2006. Political Risk and Capital Flight. Journal of International Money and Finance 25(2), 308-329. DOI: https://doi.org/10.1016/j.jimonfin.2005.11.001.

López, R.E. y Sturla, G. 2020. Híper fortunas y súper ricos: ¿Por qué un impuesto al patrimonio hace sentido? Documento de Trabajo SDT 495, Universidad de Chile. Disponible en: https://econ.uchile.cl/es/publicacion/hiper-fortunas-y-superricos-por-que-un-impuesto-al-patrimonio-hace-sentido [17 de enero 2021].

Londoño-Velez, J. y Ávila-Mahecha, J. 2018. Can Wealth Taxation Work in Developing Countries? Quasi-Experimental Evidence from Colombia. Annual Congress of the IIPF.

Mirrlees, J., Adam, S., Besley, T., Blundell, R., Bond, S., Chote, R., Gammie, M, Johnson, P., Myles, G.D. y Poterba, J. 2011. The Mirrlees Review: Conclusions and Recommendations for Reform. Fiscal Studies 32(3), 331-359.

Moretti, E. y Wilson, D.J. 2017. The Effect of State Taxes on the Geographical Location of Top Earners: Evidence from Star Scientists. American Economic Review 107(7), 1858-1903. Disponible en: https://www.aeaweb.org/articles?id=10.1257/ aer.20150508 [17 de enero 2021].

Moretti, E. y Wilson, D.J. 2019. Taxing Billionaires: Estate Taxes and the Geographical Location of the Ultra-Wealthy. National Bureau of Economic Research 26387. Disponible en: https://www.nber.org/papers/w26387 [17 de enero 2021].

OCDE 2018. The Role and Design of Net Wealth Taxes in the OECD. OECD Tax Policy Studies. Disponible en: https://www.oecd.org/ctp/the-role-and-design-of-netwealth-taxes-in-the-oecd-9789264290303-en.htm [17 de enero 2021].

OCDE 2020. Global Revenue Statistics Database. Disponible en: https://stats.oecd.org/ Index.aspx?DataSetCode=RS_GBL [1 de septiembre 2020].

Pichet, E. 2007. The Economic Consequences of the French Wealth Tax. La Revue de Droit Fiscal 14, 5. Disponible en: https://papers.ssrn.com/sol3/papers. cfm?abstract_id=1268381 [17 de enero 2021].

Piketty, T. 2014. Capital in the Twenty First Century. Cambridge, MA: Harvard University Press.

Saez, E. y Stantcheva, S. 2018. A Simpler Theory of Optimal Capital Taxation. Journal of Public Economics 162, 120-142. DOI: https://doi.org/10.1016/j. jpubeco.2017.10.004.

Saez, E. y Zucman, G. 2019a. Progressive Wealth Taxation. Brookings Papers on Economic Activity. Disponible en: https://www.brookings.edu/bpea-articles/ progressive-wealth-taxation/ [17 de enero 2021].

Saez, E. y Zucman, G. 2019b. Letter to Senator Elizabeth Warren. Disponible en: https://gabriel-zucman.eu/files/saez-zucman-wealthtax-warren.pdf [17 de enero 2021].

Seim, D. 2017. Behavioral Responses to Wealth Taxes: Evidence from Sweden. American Economic Journal: Economic Policy 9(4), 395-421. Disponible en: https://www. aeaweb.org/articles?id=10.1257/pol.20150290 [17 de enero 2021].

Scheuer, F. y Slemrod, J. 2020. Taxing Our Wealth. National Bureau of Economic Research 28150. Disponible en: https://www.nber.org/papers/w28150 [17 de enero 2021]. 
Zoutman, F.T. 2018. The Effect of Capital Taxation on Household Savings. CESifo Working Paper Series. Disponible en: https://www.cesifo-group.de/dms/ifodoc/ docs/Akad_Conf/CFP_CONF/CFP_CONF_2015/pse15-van-der-Ploeg/Papers/ Zoutman.pdf [17 de enero 2021]. EP 